\title{
Wakefield characterization in an asymmetric superconducting deflecting cavity
}

\author{
Yawei Yang* \\ Department of Engineering Physics, Tsinghua University, Beijing CN-100084, People's Republic of China \\ and Argonne National Laboratory, Argonne, Illinois 60439, USA
}

Alireza Nassiri and Geoff Waldschmidt

Argonne National Laboratory, Argonne, Illinois 60439, USA

Derun Li

Lawrence Berkeley National Laboratory, Berkeley, California 94720, USA and Department of Engineering Physics, Tsinghua University, Beijing CN-100084, People's Republic of China

\section{Huaibi Chen}

Department of Engineering Physics, Tsinghua University, Beijing CN-100084, People's Republic of China (Received 15 November 2013; published 19 March 2014)

\begin{abstract}
Nonaxially symmetric superconducting deflecting cavities are under development for a variety of purposes, but breaking the symmetry adds more complexity to wakefield characterization. This paper considers the influence of cavity asymmetry on the wakefield. Based on the TM-type deflecting cavity, we investigated how the polarized cell and the damper coupling affect the wake impedances. An improved method to characterize the wake impedances in asymmetric cavities is proposed by combining the time-domain and frequency-domain calculations. Compared to conventional methods, the new method decomposes the wake potential in terms of beam moments, which provides more information about the wakefield, and significantly reduces the simulation time with better accuracy. Using this method, we analyzed the wakefield of the deflecting cavity and the wakefield coupling between the cavities for the advanced photon source. Bench measurements were performed on a fabricated copper prototype; the measured mode field distributions and the $Q_{\text {ext }}$ values agreed well with calculations.
\end{abstract}

DOI: 10.1103/PhysRevSTAB.17.032001

PACS numbers: 29.20.Ej, 29.27.-a, 41.60.Ap

\section{INTRODUCTION}

A deflecting/crabbing cavity uses the first dipole mode for operation, which has important applications in particle accelerators. In storage-ring-based colliders or linear colliders, the deflecting cavity is designed to increase the luminosity using the crab crossing concept [1-3]. In synchrotron light sources, the deflecting cavity is considered for generating short pulse $\mathrm{x}$-ray by rf orbit deflection $[4,5]$. In addition, the deflecting cavity system is considered for delivering beams into different beamlines in some future accelerator facilities [6,7]. A superconducting (SC) deflecting cavity can economically provide a higher gradient with higher repetition rate-up to continuous wave conditions - than copper cavities, which is the requirement of these applications. The possible implementation of these

\footnotetext{
yyw721@gmail.com
}

Published by the American Physical Society under the terms of the Creative Commons Attribution 3.0 License. Further distribution of this work must maintain attribution to the author $(s)$ and the published article's title, journal citation, and DOI. applications has drawn attention to the design of the SC deflecting cavity. Wakefield characterization is a critical issue given that most superconducting deflecting cavities operate in high-current accelerator facilities.

Generally, superconducting deflecting/crabbing cavity structures are classified into two categories, TM-type and TEM-type. The TM-type deflecting cavity operates at $\mathrm{TM}_{110 y}$-like mode, which has been implemented at the KEKB B-factory $[8,9]$. The advanced photon source (APS) at Argonne National Laboratory considered using a superconducting deflecting-cavity-based scheme to generate $\mathrm{x}$-ray radiation on the order of $2 \mathrm{ps}$ or less [5]. An optimized deflecting cavity design has been proposed $[10,11]$, as shown in Fig. 1. The cavity is a squashed-cell cavity with an on-cell waveguide damper and a Y-end group on the beam pipe. The on-cell waveguide serves as a lower-order mode (LOM) damper. One of the Y-end group waveguide dampers works as an input power coupler and the other two dampers as higher-order mode (HOM) dampers. The operating mode of the TM-type deflecting cavity is not the fundamental mode, but the cavity cell geometry is relatively simple. The squashed cell cavity is 


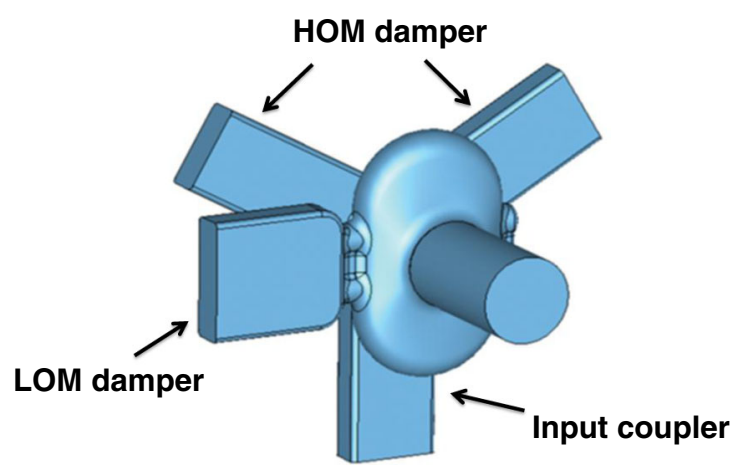

FIG. 1. The APS deflecting cavity geometry with an on-cell damper and an Y-end group on the beam pipe.

commonly adopted in a TM-type deflecting cavity. The other category is often called a TEM resonator. Most TEMtype deflecting cavities operate at the fundamental mode; the cavity geometry is more compact compared with the TM-type deflecting cavity, which makes them particularly attractive for low-frequency applications. There have been numerous designs for this type of cavity [12-14] for the possible luminosity upgrade of the LHC upgrade at CERN. The ODU/SLAC superconducting rf-dipole cavity has recently shown very promising results $[15,16]$.

Most superconducting deflecting cavity designs are nonaxially symmetric. From a cavity design point of view, breaking axial symmetry has a number of advantages. In a TM-type deflecting cavity, the squashed cell could either separate the polarizations of the dipole modes and make the damping task easier [17,18], or it could enhance the figures of merit of the deflecting mode and achieve a higher deflecting gradient [19]. In a TEM-type cavity, the highly asymmetric cavity geometry can significantly reduce the cavity transverse size and lower the surface magnetic field [20,21].

Symmetry breaking increases the difficulty in characterizing wakefields, which is very crucial given that most SC deflecting cavities operate in high-beam-current accelerator facilities. Specifically, axially symmetric geometry naturally classifies all the cavity modes into different moments [22], including monopole modes $(m=0)$, dipole modes $(m=1)$, and quadrupole modes $(m=2)$. Symmetry breaking mixes the moments, and wakefield of different moments will couple. This paper investigated how the asymmetry in cavity geometry affects the wakefield and how to characterize the wakefield in an asymmetric cavity accurately and efficiently. We focus on long-range wakefield, but short-range wakefield is discussed in several cases. In Sec. II, we discuss and present how symmetry breaking affects the wake impedance. We introduce a set of wake potential and impedance definitions based on the drive beam moments. In an asymmetric cavity, the transverse wake will be separated into polarizations, and the damper coupling has a significant effect on the wake impedance. The wake impedance cannot be directly calculated in terms of cavity mode parameters in many cases.

In Sec. III, an improved wakefield calculation method, called the hybrid method, is proposed by combining the time-domain and frequency-domain analyses. The wakefield is excited in the time domain by different drive-beam schemes. Instead of running for hundreds of meters to obtain the resolved impedance spectrum, the hybrid method only records the wake potential to a length where the broadband impedance has decayed down to a level weak enough that the narrow-band impedance peaks (the cavity resonances) can be identified. Using the residual impedance comparison, the unresolved modes can be selected, and the values of the $Q_{\mathrm{ext}} \mathrm{s}$ of the unresolved modes are calculated by the frequency-domain solver. Based on the $Q_{\text {ext }}$ values, the wake impedance spectrum can be fully resolved with better accuracy. Using the APS deflecting cavity as an example, we demonstrate that the method significantly improves the efficiency of the wakefield calculation especially in large and complex problems. The wake impedances of the single cavity, as well as the four-cavity cryomodule were calculated.

In Sec. IV, bench measurements were performed on a fabricated copper prototype of the APS deflecting cavity to verify the simulation. The agreement between the measured and simulated results of the mode field distributions and the $Q_{\text {ext }}$ values were excellent. Summaries are given in Sec. V.

\section{WAKEFIELD IN AN ASYMMETRIC DEFLECTING CAVITY}

\section{A. Wake potential and wake impedance in arbitrary cavity shape}

Wakefield is commonly characterized by wake potential and wake impedance. Wake potential is defined by the change of momentum of a test bunch following a drive bunch at a distance $s$. The test bunch travels in the same direction and at the same speed as the drive bunch $Q$. By the direction of the momentum change, wake potentials can be classified into transverse wake potentials and longitudinal wake potentials.

$$
\begin{gathered}
W_{\|}(s)=-\frac{1}{Q} \int_{0}^{L}\left[E_{\|}(z, t)\right]_{t=\frac{z+s}{c}} \mathrm{~d} z, \\
\vec{W}_{\perp}(s)=\frac{1}{Q} \int_{0}^{L}\left[\vec{E}_{\perp}+c(\hat{z} \times \vec{B})\right]_{t=\frac{z+s}{c}} \mathrm{~d} z .
\end{gathered}
$$

Generally, the drive beam can be of any length with any type of transverse distribution, and test beam does not have to follow the path of the drive beam. This adds complexity to wakefield analysis. In this paper, the drive-beam transverse distribution is decomposed in terms of unit multipole moments with respect to the beam pipe center, following the notation in Ref. [22]. In this way, we get normalized definitions of wake potentials and impedances based on the 
drive-beam moments. The normalized wake potentials and wake impedances are independent of the drive-beam parameters. The drive beam $Q$ is decomposed as

$$
Q \hat{z}=\sum_{m=0}^{\infty} C_{m} I_{m} \hat{z}
$$

where $I_{m}=q_{0} r_{0}^{m}$ is the $m$ th unit moment of the beam, $q_{0}$ is set to be $1 \mathrm{pC}$ and $r_{0}$ is set to be $1 \mathrm{~mm}$. We can normalize the wake potentials by these multipole expansions and define $W_{\|}^{m}$ and $\vec{W}_{\perp}^{m}$ as the wake potentials excited by the $m$ th unit moments of the beam $I_{m}$ :

$$
\begin{gathered}
W_{\|}^{m}(s)=-\frac{1}{I_{m}} \int_{0}^{L}\left[E_{\|}(z, t)\right]_{t=\frac{z+s}{c}} \mathrm{~d} z, \\
\vec{W}_{\perp}^{m}(s)=\frac{1}{I_{m}} \int_{0}^{L}\left[\vec{E}_{\perp}+c(\hat{z} \times \vec{B})\right]_{t=\frac{z+s}{c}} \mathrm{~d} z .
\end{gathered}
$$

Wake impedances are defined by the Fourier transform of the normalized wake potentials:

$$
\begin{aligned}
& Z_{\|}^{m}(\omega)=\mathcal{F}\left(W_{\|}^{m}\right), \\
& \overrightarrow{Z_{\perp}^{m}}(\omega)=i \mathcal{F}\left(\vec{W}_{\perp}^{m}\right) .
\end{aligned}
$$

Following this definition, $W_{\|}^{0}$ and $\vec{W}_{\perp}^{0}$ are the wake potentials excited by a unit charge $I_{0}=1 \mathrm{pC}$ passing through the beam pipe center. $W_{\|}^{1}$ and $\vec{W}_{\perp}^{1}$ are the wake potentials excited by a unit dipole charge $I_{1}=1 \mathrm{pC} \cdot \mathrm{mm}$. And wake potentials driven by any paraxial beam $Q$ could be expanded in terms of $W_{\|}^{m}$ and $\vec{W}_{\perp}^{m}$ :

$$
\begin{aligned}
& W_{\|}(s)=\frac{1}{Q} \sum_{m=0}^{\infty} C_{m} W_{\|}^{m}, \\
& \vec{W}_{\perp}(s)=\frac{1}{Q} \sum_{m=0}^{\infty} C_{m} \vec{W}_{\perp}^{m} .
\end{aligned}
$$

Note that this expansion is accurate only when the drive beam is paraxial. The coefficients $C_{m}$ of $m \geq 2$ moments are relatively weak. Thus the lower-order wake potentials dominate no matter if the cavity is symmetric or not [22].

Direct calculation in the time domain and cavity modes analysis in the frequency domain are two basic methods to calculate the long-range wakefield [23]. Direct calculation excites the wakefield by Gaussian bunches, either one bunch on the beam pipe axis (for $m=0$ longitudinal modes) or off axis (for the $m=1$ deflecting modes), passing through the cavity, and wake potential is calculated by integrating the wakefield along the beam path [24]. To resolve all the modes inside the cavity, the calculation is required to record the wake potential for hundreds of meters behind the bunch $[25,26]$. The Fourier transform of the recorded wake potential, normalized to the Gaussian bunch spectrum, gives the wake impedance. Cavity mode analysis calculates the wake potentials in the frequency domain. The wake potentials are calculated in terms of cavity mode parameters, which are the loss factors, frequencies, and external quality factors.

\section{B. Polarization and wake impedance}

Breaking axial symmetry increases the difficulty in wakefield analysis. In an axially symmetric cavity, the cavity modes are naturally separated into different moments. $W_{\|}^{0}$ only couples with the monopole modes in the cavity, and $\vec{W}_{\perp}^{1}$ only couples with the dipole modes in the cavity. The radial dependence of the wake impedances makes them very simple expressions [27]. However, in a nonaxially symmetric cavity, the cavity modes cannot be classified into different momentums.

We demonstrate the polarization effects on the wake impedance using TM-type deflecting cavities as models. The transverse profile of a TM-type cavity is either elliptical or racetrack shaped. We selected an elliptical cavity in this analysis. The effects of the polarized shape on wake impedances were examined by adjusting the axial ratio of long axes $a$ to short axes $b$, as shown in Fig. 2. In this case, the horizontal and vertical symmetry were still maintained while the axial symmetry was broken. The analysis involved direct calculation using CST PARTICLE STUDIO in the time domain and cavity mode analysis using CST Microwave Studio in the frequency domain. To account for the damping effect in a simplified way, a lossy metal skin was applied to the cavity cell surface to absorb the wakefield energy generated by beam.

Different beam excitation schemes were used [24,26], as shown in Fig. 3. According to Eqs. (3a), (3b), the longitudinal wake $W_{\|}^{0}$ was calculated by launching a single Gaussian beam on axis. The transverse wake $\vec{W}_{\perp}^{1}$ was

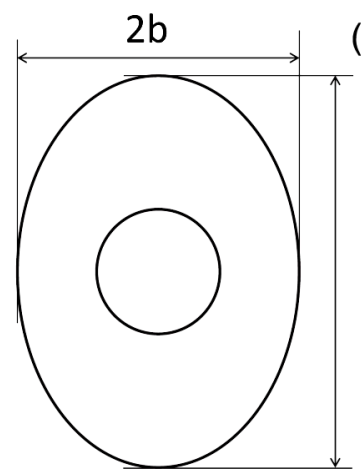

(a)

(b)

FIG. 2. Elliptical cavity used for investigating the polarized shape effect on wake impedance: (a) transverse profile of the elliptical cavity, the axial ratio is defined as the ratio of long axes $a$ to short axes $b$; (b) cavity model with lossy surface to account for the damping effect. 


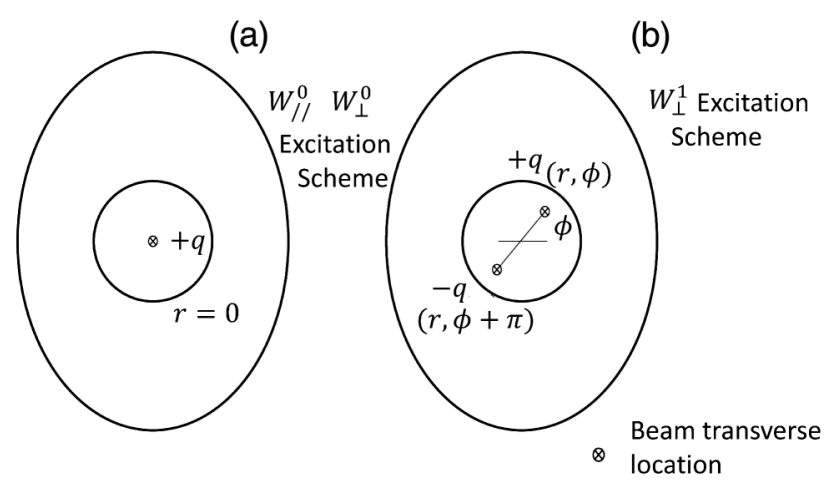

FIG. 3. Different beam excitation schemes for wakefield calculation: (a) single-beam scheme for $\vec{W}_{\|}^{0}$; (b) dipole beam scheme for $\vec{W}_{\perp}^{1} \cdot r$ is set in the range of $R / 30$ to $R / 10$, and the wake impedance dependence on the angle $\phi$ needs to be investigated in the asymmetric cavity.

calculated by launching two beams, which were set off axis with a radius of $r$ with respect to the beam pipe center in opposite directions. The frequency resolution is determined by the recorded length of the wakefield. And the frequency range of the wake impedance is determined by the Gaussian bunch rms length. The advantage of the two-beam scheme over the one-beam scheme for $\vec{W}_{\perp}^{1}$ calculation is that the two-beam scheme has dominant dipole moments, while the off-axis one-beam scheme still has a strong monopole moment. In our simulation, $r$ was set in the range of $R / 30$ to $R / 10$. Several calculations with different values of $r$ were performed to check the consistency. The angle $\phi$ determined the dipole excitation orientation.

\section{Longitudinal wake}

In an axially symmetric cavity, the longitudinal wake potential and wake impedance can be expressed as the superposition of monopole modes:

$$
\begin{gathered}
W_{\|}^{0}(s)=\sum_{n} 2 k_{\| n} \cos \frac{\omega_{\| n} s}{c} \exp \left(-\frac{\pi s}{Q_{n} \lambda_{n}}\right), \\
Z_{\|}^{0}(\omega)=\sum_{n} \frac{\frac{1}{2}\left(\frac{r}{Q}\right)_{\| n} Q_{n}}{1+i Q_{n}\left(\frac{\omega_{n}}{\omega}-\frac{\omega}{\omega_{n}}\right)},
\end{gathered}
$$

where $(r / Q)_{\| n}$ and angular frequency $\omega_{n}$ are the $n$th monopole mode parameters, and $k_{\| n}=\frac{1}{4}\left(\frac{r}{Q}\right)_{\|} \omega_{n}$ is the loss factor.

Figure 4 compares the longitudinal wake impedance in a symmetric cavity and an elliptical cavity $(a=1.75 b)$. The elliptical cavity geometry is based on the optimization of the APS deflecting cavity. The frequency-domain results are calculated using Eq. (7). The time-domain results are based on Fourier transform of longitudinal wake potential $W_{\|}^{0}$, which was calculated launching a single Gaussian beam on axis. To normalize the frequency, the horizontal axis is set as a size-independent quantity $k d$, where $k$ is the
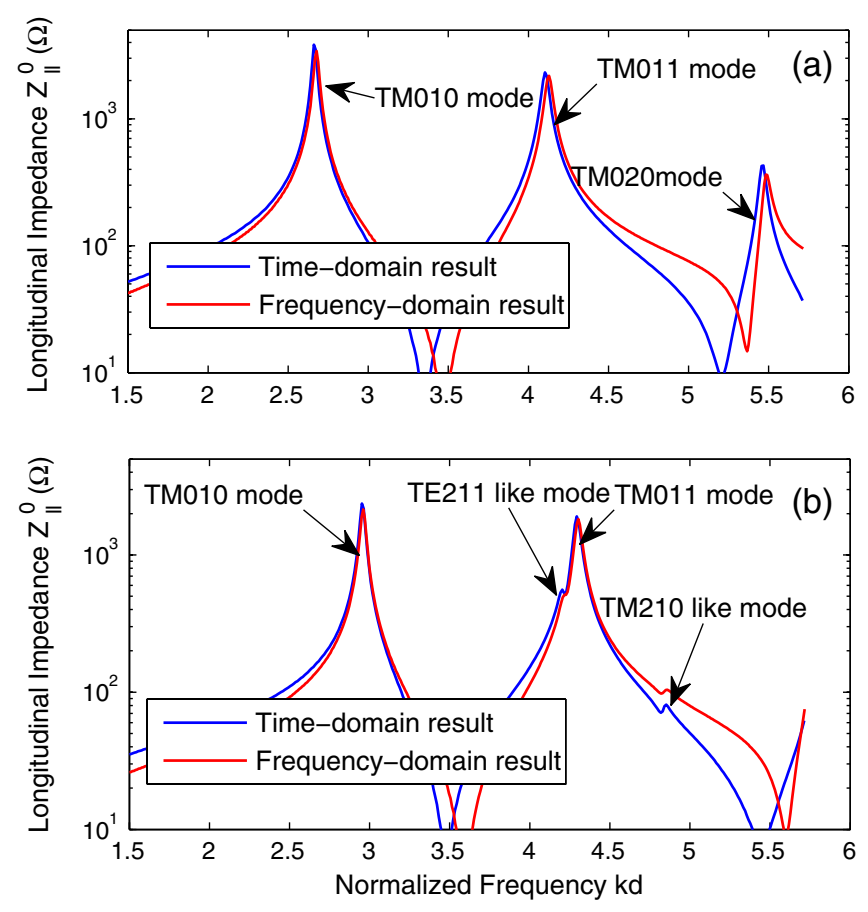

FIG. 4. Longitudinal wake impedances $Z_{\|}^{0}$ from time-domain and frequency-domain calculations: (a) The longitudinal wake impedance in a symmetric cavity; (b) The longitudinal wake impedance in an elliptical cavity $(a=1.75 b)$. The horizontal axis is the normalized frequency $k d$.

wave number and $d$ is set to be the average of the long axis $a$ and short axis $b$.

As Fig. 4 shows, there are quadrupolelike modes present in longitudinal impedance. This is due to the fact that the quadrupole modes will be compressed in a highly polarized elliptical cavity and the modes have a longitudinal electrical field along the cavity center. Even if the beam passes through the elliptical cavity center, the quadrupolelike modes are excited. Thus the impedance peaks of quadrupolelike modes are seen in the longitudinal impedance spectrum.

The time-domain results (blue curves) and frequencydomain results (red curves) are consistent around the impedance peaks. Equation (7) can still be used to calculate the longitudinal impedance $Z_{\|}^{0}$ in an asymmetric cavity. But more modes need to be taken into account, such as the quadrupolelike modes. There is inconsistency between time-domain and frequency-domain results when the impedance is low. Two factors are believed to cause this inconsistency. One is the broadband impedance effect, which is included in the timedomain calculation but not in the frequency-domain analysis. The other effect is that the wake spectrum at the high frequency is affected by the cavity modes at higher frequencies, which is not considered in the frequency-domain analysis.

\section{Transverse wake}

The polarization has a significant effect on the transverse wake. In an axially symmetric cavity, the transverse wake 
potential $\vec{W}_{\perp}^{1}$ can be expressed as the superposition of dipole modes:

$$
\vec{W}_{\perp}^{1}(s)=\hat{r} \sum_{n} 2 k_{\perp n} \sin \frac{\omega_{\perp n} s}{c} \exp \left(-\frac{\pi s}{Q_{n} \lambda_{n}}\right),
$$

where $\hat{r}$ is the drive dipole orientation, and $k_{\perp n}$ is the transverse loss factor of the $n$th dipole mode, which is defined as

$$
k_{\perp n}=\frac{1}{4}\left(\frac{r}{Q}\right)_{\perp n}\left(\frac{\omega_{n}}{c}\right) \omega_{n} ;
$$

here $(r / Q)_{\perp n}$, wave number $\omega_{n} / c$, and angular frequency $\omega_{n}$ are the $n$th dipole mode parameters. The transverse wake direction is determined by the drive dipole orientation. The transverse wake impedance can be calculated by taking the Fourier transform of the transverse wake potential:

$$
\vec{Z}_{\perp}^{1}(\omega)=\hat{r} \sum_{n} \frac{\frac{1}{2}\left(\frac{r}{Q}\right)_{\perp n} \frac{\omega_{n}}{c} Q_{n}}{1+i Q_{n}\left(\frac{\omega_{n}}{\omega}-\frac{\omega}{\omega_{n}}\right)} .
$$

The transverse impedance becomes polarized after breaking symmetry. The transverse impedance is independent of the varying angle $\phi$ in an axially symmetric cavity. However, in an elliptical cavity, the dipole modes are separated into horizontal and vertical polarizations. The transverse impedance is different with different angle $\phi$. There is no general way to calculate the transverse wake impedances in terms of cavity mode parameters. However, this calculation can be performed in some special cases, when the cavity mode field pattern in a transverse cross section is constant along the longitudinal direction [28], which means the longitudinal component of the mode vector $\vec{A}_{n}$ can be written as

$$
A_{n z}(x, y, z)=f_{n}(x, y) g(z) .
$$

When Eq. (11) is satisfied, the transverse wake potential can be expressed as

$$
\vec{W}_{\perp}^{1}(s)=\sum_{n} 2 k_{\perp n} \cos \theta \sin \frac{\omega_{\perp n} s}{c} \exp \left(-\frac{\pi s}{Q_{n} \lambda_{n}}\right) \hat{r}_{n},
$$

where $\theta$ is the angle between the excitation dipole transverse azimuthal angle $\phi$ and the polarization direction $\hat{r_{n}}$. Note that the transverse wake direction is not determined by the drive dipole azimuthal angles, but by the cavity mode polarization directions. The cavity modes below the cutoff frequency are of great importance in the wakefield damping study. Equation (11) is approximately valid for these modes in an elliptical cavity. The dipolelike modes in an elliptical cavity have two polarizations in the horizontal $x$ and vertical $y$ planes. We decompose the drive dipole $I_{1} \hat{r}$ into these two planes:

$$
I_{1} \hat{r}=I_{x} \hat{x}+I_{y} \hat{y}=q x_{0} \hat{x}+q y_{0} \hat{y} .
$$

Using Eq. (12), the transverse wake potential $\vec{W}_{\perp}^{1}$ excited by the drive dipole $I_{1} \hat{r}$ is expressed as

$$
\vec{W}_{\perp}^{1}(s)=I_{x} W_{x}^{1} \hat{x}+I_{y} W_{y}^{1} \hat{y},
$$

and the transverse wake impedance can be calculated as

$$
\vec{Z}_{\perp}^{1}=Z_{x}^{1} \cos \theta \hat{x}+Z_{y}^{1} \sin \theta \hat{y},
$$

where $Z_{x}^{1}$ and $Z_{y}^{1}$ can be expressed as a similar form as in an axially symmetric cavity:

$$
\begin{aligned}
& \vec{Z}_{x}^{1}(\omega)=\hat{x} \sum_{n x} \frac{\frac{1}{2}\left(\frac{r}{Q}\right)_{x} \frac{\omega_{n x}}{c} Q_{n x}}{1+i Q_{n x}\left(\frac{\omega_{n x}}{\omega}-\frac{\omega}{\omega_{n x}}\right)}, \\
& \vec{Z}_{y}^{1}(\omega)=\hat{y} \sum_{n y} \frac{\frac{1}{2}\left(\frac{r}{Q}\right)_{y} \frac{\omega_{n y}}{c} Q_{n y}}{1+i Q_{n y}\left(\frac{\omega_{n y}}{\omega}-\frac{\omega}{\omega_{n y}}\right)} .
\end{aligned}
$$

However, above the cutoff frequency, the cavity dipole modes will couple with the beam pipe modes. The decomposition will become inaccurate for these modes. Figure 5 compares the transverse impedance in different transverse planes of an elliptical cavity with $(a=1.75 b)$. The time-domain results were calculated from the Fourier transform of the recorded wake potential, and the frequency-domain results were based on Eqs. (15) and (16a), (16b). The impedances are compared in three different planes, the horizontal plane $\left(\phi=0^{\circ}\right)$, the vertical plane $\left(\phi=90^{\circ}\right)$, and the $\phi=60^{\circ}$ plane. The traverse impedance at different azimuthal angle $\phi$ is different, but it can be approximately decomposed using Eqs. (16a), (16b). In either plane, the directly calculated impedance (blue line) and cavity-mode-derived impedance (cyan line) are quite consistent at the impedance peaks but are significantly inconsistent when the impedance is low, especially at low frequency. The inconsistency is mainly due to broadband impedance; a simple estimate is [22]

$$
\overrightarrow{Z_{\perp}^{1}} \approx 60 \Omega \times \frac{1}{b} \hat{r}, \quad \omega<\frac{c}{b} .
$$

Note the equation above is valid when $k r_{b}<1$, where $k$ is the wave number and $r_{b}$ is the beam pipe radius. As shown in Fig. 5, the impedance magnitudes from the time domain (blue line) and the frequency domain (red line) are quite consistent in three different planes after taking into account the broadband impedance using Eq. (17). With Eqs. (15) and (16a), (16b), the transverse wake in any transverse plane can be decomposed into the polarization planes, which provides a very effective way to investigate the transverse wake in an asymmetric cavity. However, above the beam pipe cutoff frequency, the cavity mode 

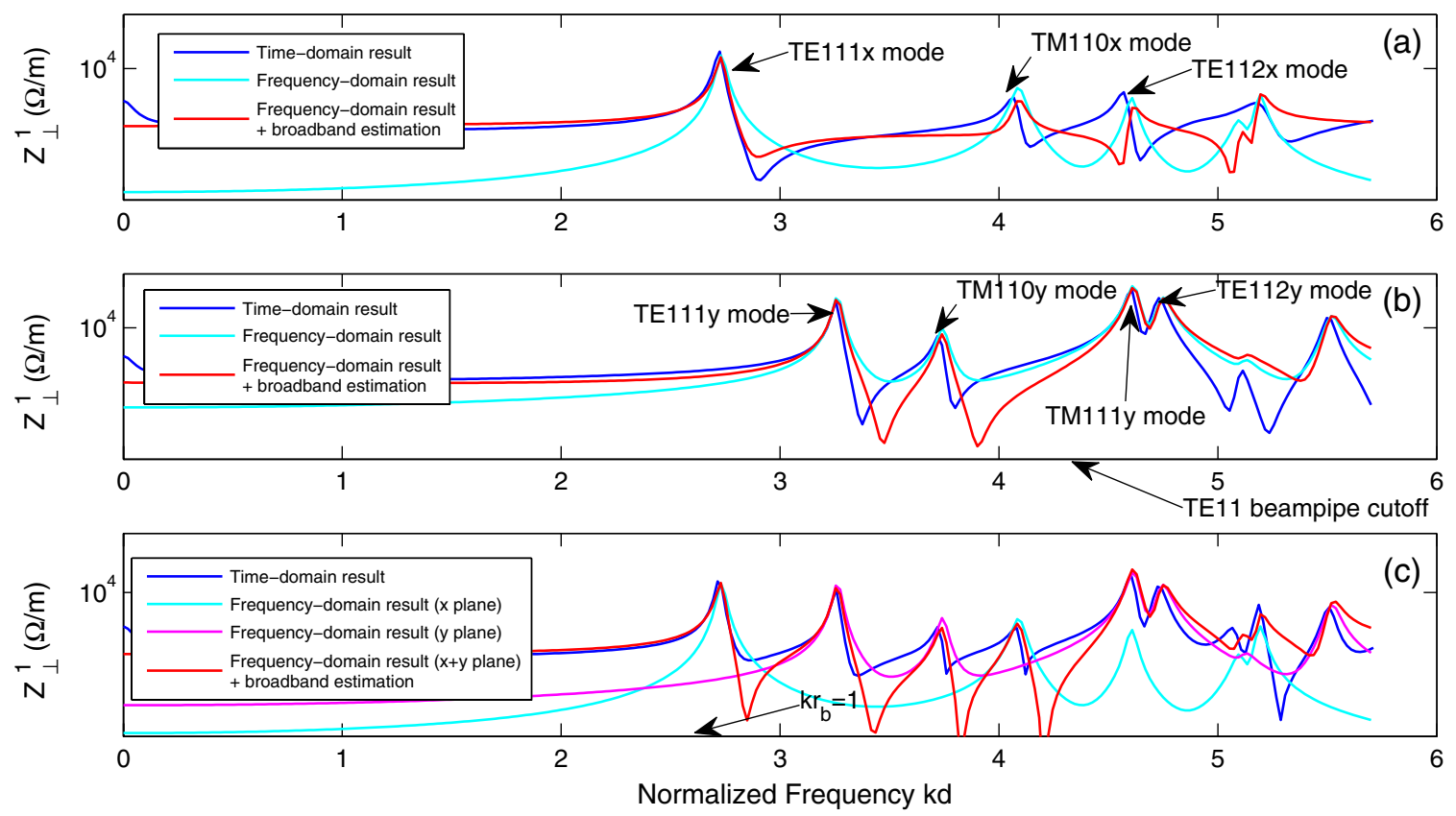

FIG. 5. Transverse wake impedances $Z_{\perp}^{1}$ in an elliptical cavity $(a=1.75 b)$ at different transverse planes: (a) the horizontal plane; (b) the vertical plane; (c) the $\phi=60^{\circ}$ plane. In each figure, three impedance curves are compared: directly calculated impedance (blue curve), cavity-mode derived impedance (cyan or pink curve), and cavity mode derived impedance plus the broadband estimation (red curve).

polarization directions will change along the beam path, Eqs. (16a), (16b) is no longer valid any more. Thus there is significant inconsistency around $k d=5$ in Fig. 5. In this case, the frequency-domain method is not valid and more azimuthal angles $\phi$ are required in the direct calculations.

\section{Transverse and longitudinal wake coupling at asymmetric modes}

In Sec. II B, the wake impedance of the elliptical cavity was investigated. Even though the elliptical-cell cavity is not axially symmetric, both the vertical symmetry and horizontal symmetry are maintained and the cavity still has a geometric center. The cavity modes in such cavities can be classified into longitudinal modes and transverse modes. As long as the beam passes through the cavity center, each mode has only one kick effect, either in the transverse or the longitudinal direction. This fact is not true in an asymmetric cavity when the cavity vertical and horizontal symmetry are broken and the cavity modes become asymmetric with respect to the cavity center. In the superconducting deflecting cavity, this happens when the cavity cell becomes highly asymmetric, such as the BNL quarter wave cavity [12] and, more commonly, when a damper is added. In the APS deflecting cavity shown in Fig. 1, both the on-cell damper and the Y-end group will push cavity modes off center, which makes these modes asymmetric. This effect on the wakefield is significant. We will demonstrate this effect using the on-cell damper in the APS deflecting cavity as an example.

The on-cell damper [29], which is adopted in the APS deflecting cavity, is designed to provide very strong coupling for the $\mathrm{TM}_{010}$-like mode. The operating current of the APS deflecting cavity is about $150 \mathrm{~mA}$, which will induce a beam loss power over $1 \mathrm{~kW}$ at this mode. The on-cell damper can provide the required strong damping for the $\mathrm{TM}_{010}$-like mode.

\section{Transverse wake induced by asymmetric longitudinal mode}

A strong transverse wake is present after the on-cell damper is added. As shown in Fig. 6, both the longitudinal wake (blue curve) and the transverse wake (green curve) can be excited by a single Gaussian bunch (the rms length $=25 \mathrm{~mm}$ ), the profile of which is shown as the red curve, passing through the center of the deflecting cavity with an on-cell damper. Spectrum analysis has shown that both the longitudinal and transverse wake impedance is dominated by the $\mathrm{TM}_{010}$-like mode, as shown in Fig. 7. Compared to the dipole-mode excited transverse wake, this transverse wake is less dependent on the offset radius, which can be classified as $W_{\perp}^{0}$.

The existence of $W_{\perp}^{0}$ at the $\mathrm{TM}_{010}$-like mode is caused by the asymmetric coupling of the on-cell damper. As shown in Fig. 8, the $\mathrm{TM}_{010}$-like mode center will be offset due to the on-cell damper. The coupling of the on-cell 


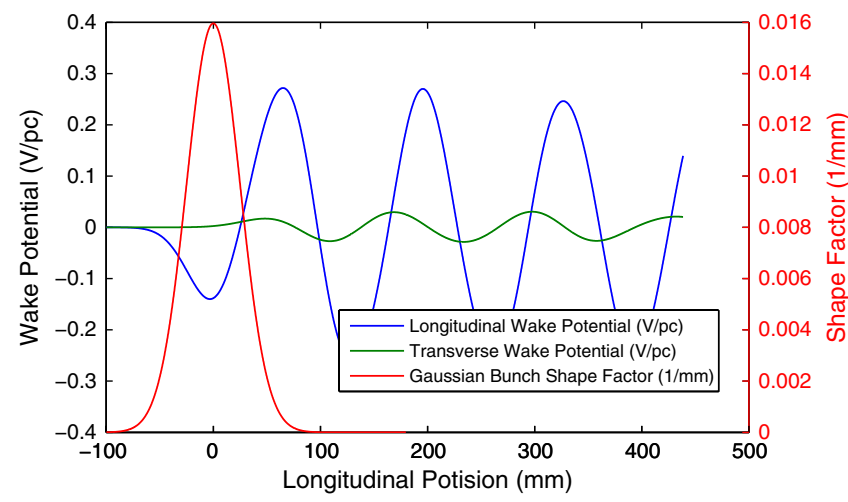

FIG. 6. Gaussian drive bunch distribution and excited longitudinal and transverse wake potential. The rms length of the bunch is $25 \mathrm{~mm}$.
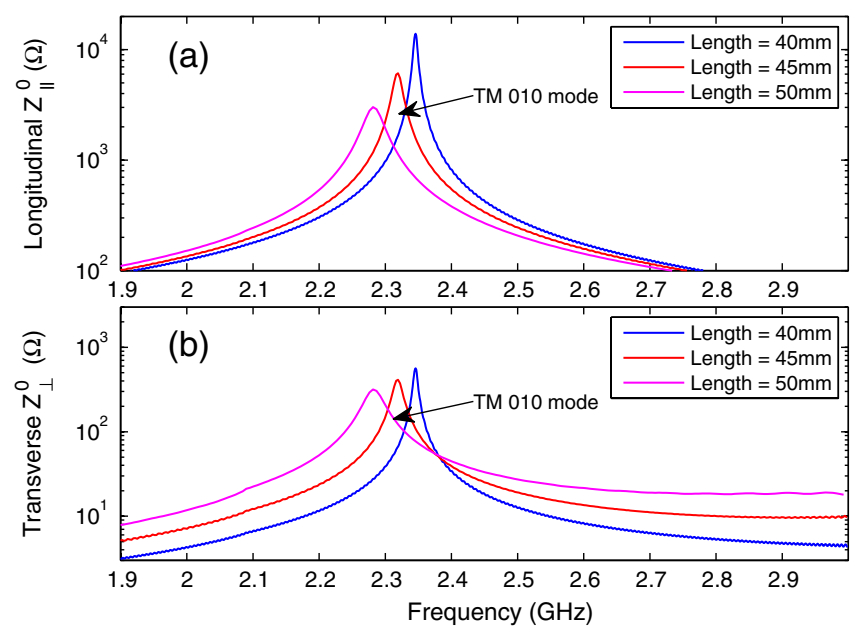

FIG. 7. The longitudinal wake $W_{\|}^{0}$ spectrum (a) and transverse wake $W_{\perp}^{0}$ spectrum (b) with different on-cell opening length $L$. We can clearly see the peaks of $\mathrm{TM}_{010}$-like mode.

damper is mainly tuned by the on-cell opening length $L$. As $L$ increases, the coupling becomes stronger. Around the cavity center, both the amplitude and phase of the longitudinal electric field $E_{z 0} \exp (i \phi)$ have transverse gradients along the damper coupling direction $x$. We expand the $\mathrm{TM}_{010}$-like longitudinal electrical field spatially as

$$
E_{z}=\left[E_{z 0}+\frac{\partial E_{z 0}}{\partial x} x\right] \exp \left(i \frac{\partial \phi}{\partial x} x\right)
$$

where the transverse gradients are expressed in terms of $\frac{\partial E_{z 0}}{\partial x}$ and $\frac{\partial \phi}{\partial x}$. Consider the $\mathrm{TM}_{010}$-like mode only in the wakefield analysis:

$$
W_{\|}^{0}(s)=-\int_{0}^{L}\left[E_{z}(z, t)\right]_{t=\frac{z+s}{c}} \mathrm{~d} z
$$

Using Eq. (6), $W_{\|}^{0}(s)$ is expressed as

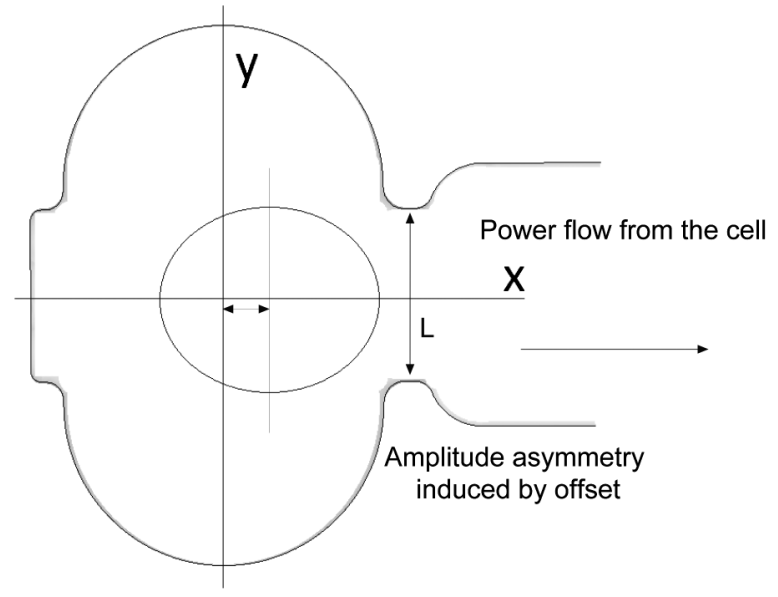

FIG. 8. The $\mathrm{TM}_{010}$-like mode longitudinal electrical field amplitude asymmetry due to the on-cell damper coupling. The coupling is tuned by the on-cell opening length $L$.

$$
W_{\|}^{0}(s)=k_{0} \cos \frac{\omega_{0} s}{c} \exp \left(-\frac{\pi s}{Q_{\text {ext }} \lambda_{0}}\right), \quad(s>0),
$$

where $k_{0}, \omega_{0}, Q_{\text {ext }}$, and $\lambda_{0}$ are parameters of the $\mathrm{TM}_{010}$-like modes. According to the Panofsky-Wenzel theorem:

$$
\vec{\nabla}_{\perp} W_{\|}^{0}(s)=\frac{\partial \vec{W}_{\perp}^{0}(s)}{\partial s}
$$

we can obtain the transverse kick

$$
\frac{\partial W_{\perp}^{0}(s)}{\partial s}=\frac{\partial E_{z 0}}{\partial x} \frac{W_{\|}^{0}(s)}{E_{z 0}}+\frac{\partial \phi}{\partial x} W_{\|}^{0}(s) i
$$

Assuming $\frac{\partial E_{z 0}}{\partial x} \frac{1}{E_{z 0}}$ and $\frac{\partial \phi}{\partial x}$ are constant along the beam path, we can get the expression for the transverse wake using the integral of Eq. (22) along the beam path and obtain

$$
\begin{aligned}
{\overrightarrow{W_{\perp}^{0}}}_{\perp}(s)= & {\left[\frac{c}{\omega} \frac{\partial \phi}{\partial x} k_{0} \cos \frac{\omega_{0} s}{c}+\frac{c}{\omega_{0}} \frac{E_{z 0}}{x} \frac{1}{E_{z 0}} k_{0} \sin \frac{\omega_{0} s}{c}\right] } \\
& \times \exp \left(-\frac{\pi s}{Q_{0} \lambda_{0}}\right) \hat{x} .
\end{aligned}
$$

We define the damper kick factor $\kappa$, which is the ratio of the transverse wake potential to the longitudinal wake potential, and $\theta$, which is the phase difference between the transverse and longitudinal wake potentials:

$$
\kappa=\sqrt{\frac{c}{\omega}\left[\left(\frac{\partial \phi}{\partial x}\right)^{2}+\left(\frac{\partial E_{z 0}}{\partial x} \frac{1}{E_{z 0}}\right)^{2}\right]},
$$


TABLE I. The $\kappa$ and $\theta$ from time-domain and frequency-domain calculations with different coupling strengths through the on-cell damper.

\begin{tabular}{|c|c|c|c|c|c|c|c|}
\hline \multirow[b]{2}{*}{ Opening $L(\mathrm{~mm})$} & \multirow[b]{2}{*}{$\begin{array}{c}Q_{\text {ext }} \\
(\text { Omega3P) }\end{array}$} & \multicolumn{3}{|c|}{ Formula-derived result } & \multicolumn{3}{|c|}{ Direct simulation } \\
\hline & & $\begin{array}{c}\frac{\partial E_{z 0}}{\partial x} \frac{1}{E_{z 0}}\left(\mathrm{~mm}^{-1}\right) \\
(\text { Omega3P })\end{array}$ & $\begin{array}{c}\frac{\partial \phi}{\partial x} \\
(\text { Omega3P) }\end{array}$ & $\begin{array}{c}\kappa \\
(\text { Eq. } 24)\end{array}$ & $\begin{array}{c}\theta \\
\text { (Eq. 25) }\end{array}$ & $\begin{array}{c}\kappa \\
(\mathrm{CST} \text { T-solver) }\end{array}$ & $\begin{array}{c}\theta \\
\text { (CST T-solver) }\end{array}$ \\
\hline 35 & 1015.2 & $9.98 \times 10^{-4}$ & $9.00 \times 10^{-5}$ & 0.0229 & $-84.851^{\circ}$ & 0.0228 & $-84.97^{\circ}$ \\
\hline 40 & 384.4 & $1.79 \times 10^{-3}$ & $2.46 \times 10^{-4}$ & 0.0413 & $-82.159^{\circ}$ & 0.0405 & $-82.8^{\circ}$ \\
\hline 45 & 168.3 & $2.92 \times 10^{-3}$ & $5.19 \times 10^{-4}$ & 0.0676 & $-79.904^{\circ}$ & 0.0677 & $-80.25^{\circ}$ \\
\hline 50 & 86.3 & $4.49 \times 10^{-3}$ & $1.02 \times 10^{-3}$ & 0.1052 & $-77.18^{\circ}$ & 0.1045 & $-77.19^{\circ}$ \\
\hline 55 & 61.3 & $7.01 \times 10^{-3}$ & $1.823 \times 10^{-3}$ & 0.1653 & $-75.41^{\circ}$ & 0.1601 & $-75.62^{\circ}$ \\
\hline
\end{tabular}

$$
\theta=-\arctan \left(\frac{\frac{\partial E_{z 0}}{\partial x} \frac{1}{E_{z 0}}}{\frac{\partial \phi}{\partial x}}\right)
$$

And the transverse wake can be expressed as

$$
\vec{W}_{\perp}^{0}(s)=\kappa W_{\|}(s) \exp (i \theta) \hat{x}
$$

Equation (26) relates the transverse wake to the amplitude and phase gradients of the $\mathrm{TM}_{010}$-like mode, which can be calculated using the ACE3P Omega3P solver [30]. Table I compares the calculated frequency-domain values of $\kappa$ and $\theta$ using the Omega3P solver and the time-domain results using CST Particle Studio.

We can see both $\kappa$ and $\theta$ values from frequency-domain calculations agree very well with the time-domain simulations. As the on-cell opening increases, the transverse gradients of the magnitude and phase of the electrical field grow as the coupling becomes stronger. Thus the transverse wake becomes stronger. The amplitude gradient plays a more significant role than the phase gradient. The strong transverse wake induced by the on-cell damper is very similar to the coupler-induced kick in the linac $[31,32]$. Compared with the linac coupler kick, the ratio of the transverse kick to longitudinal kick is much stronger in the deflecting cavity with an on-cell damper due to the strong coupling through the damper. The $\kappa$ can be as high as 0.16 . One thing to note is that the analysis above is based on the assumption $\frac{\partial E_{z 0}}{\partial x} \frac{1}{E_{z 0}}$ and $\frac{\partial \phi}{\partial x}$ are constant, which is not true in many cases. In those cases, the transverse wake can not be expressed simply in terms of cavity mode parameters. Direct calculation is the appropriate way to investigate the longitudinal and transverse coupling in those cases.

\section{Short-range effect}

Both short-range and long-range effects of $W_{\perp}^{0}(s)$ are investigated for the APS deflecting cavity. To characterize the short-range self-kick effect, a damper kick factor is defined by the transverse moments received by a relativistic Gaussian beam going through the cavity center:

$$
k_{\perp 0}=\int_{-6 \sigma}^{6 \sigma} \lambda(s) W_{\perp}^{0} \mathrm{~d} s,
$$

where $\sigma$ is the rms length of the Gaussian beam, and $\lambda(s)$ is the bunch distribution function. Note that $W_{\perp}^{0}$ is the transverse wake excited by the single beam passing through the cavity center. Thus the unit of the coupler kick factor is $\mathrm{V} / \mathrm{pC}$, which is different from the unit of the conventional kick factor $\mathrm{V} / \mathrm{pC} / \mathrm{m}$. As Eq. (26) shows, the transverse wake $W_{\perp}^{0}$ is not strongly related to the drive beam offset.

The loss factor and damper kick factor can be directly calculated based on the $W_{\perp}^{0}$ and $W_{\|}^{0}$ with CST Particle Studio. And the $W_{\perp}^{0}$ and $W_{\|}^{0}$ are excited with a single Gaussian bunch passing through the on-cell damping cavity center. Table II shows the calculated results with different bunch rms lengths. Two coupling strengths are investigated, one at $Q_{\text {ext }}=384$ and the other at $Q_{\text {ext }}=84$. In either case, the longitudinal loss factor drops as the bunch length gets longer, while the transverse kick factor increases as the bunch length gets longer.

In the advanced photon source, the rms bunch length is about $10 \mathrm{~mm}$. As Table II shows, the kick factor is relatively weak at this bunch length. Thus the short-range effect is not likely to induce beam instability.

TABLE II. Calculated loss factor and damping kick factor with different Gaussian-bunch rms lengths. Two coupling strengths $Q_{\text {ext }}=86$ and $Q_{\text {ext }}=384$ are investigated with CST Particle Studio.

\begin{tabular}{lccccc}
\hline \hline & \multicolumn{2}{c}{$\begin{array}{c}Q_{\text {ext }}=384 \\
(L=40 \mathrm{~mm})\end{array}$} & & \multicolumn{2}{c}{$\begin{array}{c}Q_{\text {ext }}=86 \\
(L=50 \mathrm{~mm})\end{array}$} \\
\cline { 2 - 3 } \cline { 6 - 7 } & $\begin{array}{c}\text { Loss } \\
\text { factor }\end{array}$ & $\begin{array}{c}\text { Kick } \\
\text { factor }\end{array}$ & & $\begin{array}{c}\text { Loss } \\
\text { factor }\end{array}$ & $\begin{array}{c}\text { Kick } \\
\text { factor } \\
\text { Bunch rms }\end{array}$ \\
length $(\mathrm{mm})$ & $(\mathrm{V} / \mathrm{nC})$ & $(\mathrm{V} / \mathrm{nC})$ & & $(\mathrm{V} / \mathrm{nC})$ & $(\mathrm{V} / \mathrm{nC})$ \\
\hline 5 & 544.4 & 0.006 & & 522.5 & 0.006 \\
10 & 288.2 & 0.225 & & 288.2 & 0.215 \\
15 & 170.1 & 1.161 & & 178.2 & 1.372 \\
20 & 105.2 & 2.285 & & 109.1 & 3.171 \\
25 & 59.92 & 2.768 & & 63.41 & 4.492 \\
30 & 29.08 & 2.684 & & 31.24 & 4.681 \\
35 & 12.07 & 2.342 & & 12.82 & 4.106 \\
\hline \hline
\end{tabular}




\section{Long-range effect}

Based on the Eqs. (20) and (26), we only consider the $\mathrm{TM}_{010}$-like mode, and express the damper induced transverse wake as

$$
\vec{W}_{\perp}^{0}(s)=\kappa k_{0} \exp \left(-\frac{\pi s}{Q_{\text {ext }} \lambda_{0}}\right) \cos \left(\frac{\omega_{0} s}{c}+\theta\right) \hat{x}
$$

where $k_{0}$ is the loss factor of the $\mathrm{TM}_{010}$-like mode. As the damper coupling gets stronger, the phase gradient and amplitude gradient increase, which causes stronger transverse wake. On the other hand, as the on-cell opening gets larger, the $\mathrm{TM}_{010}$-like mode decays at a much faster rate. Using Eq. (28), we calculated how the transverse wake potential envelopes decays with different values of $Q_{\text {ext }}$ of the on-cell damper coupling.

As shown in Fig. 9, at a distance no larger than several meters, the transverse wake envelope becomes stronger as the coupling gets stronger. However, the transverse wake at a distance greater than 5 meters drops as the coupling get stronger due to a faster damping rate.

In the APS standard operation mode [33], there are 24 bunches in the storage ring, and the distance between the bunches is $45.86 \mathrm{~m}$. This distance is far enough that we should make the coupling as strong as possible to reduce the transverse kick on the following bunches. However, in some special operation modes, when the intrabunch distance is much shorter, the transverse kick might cause beam emittance degrading and beam instability.

An easy way to alleviate the transverse kick $W_{\perp}^{0}$ can be achieved by an appropriate configuration of the LOM coupler direction. In the current scheme, two groups of deflecting cavities are required to be inserted into the storage ring. In each group, there is a four-cavity cryomodule. The transverse kicks can cancel each other by placing the LOM damper alternately in opposite directions.

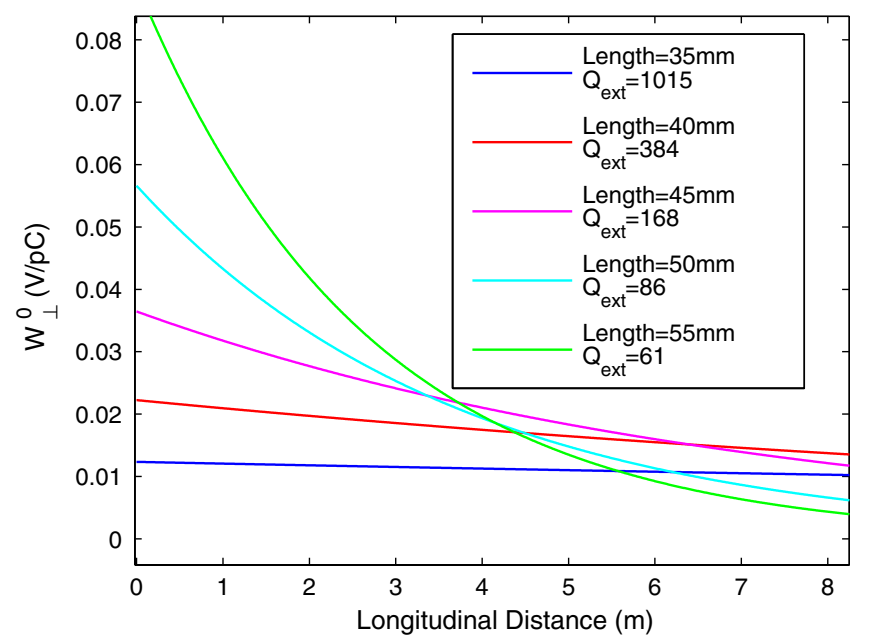

FIG. 9. Transverse wake potential $W_{\perp}^{0}$ envelopes with different on-cell damper coupling strengths.
The first cavity has the on-cell damper on the left, while the second cavity has the damper on the right. Thus the kick effect can cancel each other.

\section{THE HYBRID METHOD FOR WAKEFIELD CALCULATION}

\section{A. Hybrid wakefield analysis steps}

Both the direct calculation and the cavity mode analysis have their pros and cons. The direct method calculates how the beam excites the wakefield. There have been a number of 3D time-domain codes developed for wakefield simulation, including CST PARTICle StUdio, GdFidL, and ACE3P T3P solver. The beam-cavity interaction can be precisely simulated with these codes. However, the wakefields need to be recorded to a length of hundreds meters or several kilometers to fully resolve the high- $Q_{\text {ext }}$ cavity modes, which is very time consuming. To reduce the calculation time, there have been a number of ways developed [34,35], which use a yet unresolved impedance spectrum to evaluate the resolved impedance. However, these methods are effective only when the wakefields has been recorded to a length that the impedance is partially resolved. In the example in Ref. [35], a 600 meter calculation is required to resolve some cavity modes with $Q_{\text {ext }} \approx 3 \times 10^{4}$.

Cavity mode analysis is also an effective way to calculate the wake impedance in terms of cavity mode parameters in the frequency domain. Using advanced parallel codes like ACE3P OMEGA3P solver, the simulations are very fast and accurate [30]. However, in many asymmetric cavities, the longitudinal and transverse wake cannot be directly calculated in terms of the values of $r / Q$ and $Q_{\text {ext }}$, and it is tedious to iterate the calculation for many modes.

We developed an improved method, named the hybrid method, to take advantage of both methods to improve the accuracy and speed further. There are three main steps:

(i) Time-domain excitation: We first use the time-domain method to calculate the longitudinal and transverse wakes to a certain length, where the broadband impedance and low- $Q_{\text {ext }}$ cavity modes are resolved while the high- $Q_{\text {ext }}$ cavity modes are not resolved. As is well known, the broadband component of the wakefield decays at a much faster speed compared to the cavity mode resonances. In the conventional method, the simulation needs to record the wake potential to a length that all the high- $Q_{\text {ext }}$ cavity modes are resolved or partially resolved. If we only need to get resolved impedance spectrum for the broadband component and low- $Q_{\text {ext }}$ cavity modes, the recorded length of wake potentials can be much shorter and the simulation can be much faster.

(ii) Residual impedance comparison: To determine whether the impedance at a cavity mode is resolved or not for a certain mode, $\mathrm{Li}$ and Rimmer [25] proposed a method using impedance comparison. An improved method is used based on this method. Assuming we 
calculate the wake potential to a length $L$, we select two distances, $L 1$ and $L 2$. $L 1$ is chosen based on whether the broadband component of the wake has decayed down to a weaker level than the cavity mode impedance. $L 2$ is typically the average of $L 1$ and $L$. In this way, two residual impedances can be calculated: $Z_{L 2}^{L}=\frac{1}{c} \int_{L 2}^{L} w(s) e^{i \omega_{n c} \frac{s}{c}} d s$ and $Z_{L 1}^{L}=\frac{1}{c} \int_{L 1}^{L} w(s) e^{i \omega_{n} \frac{s}{c}} d s$, where $L 1<L 2<L$. To determine if a cavity mode spectrum is resolved or not, a resolution parameter $e$ is defined as the impedance ratio of $Z_{L 2}$ and $Z_{L 1}$ at a cavity mode $\omega_{n}$ :

$$
\begin{aligned}
e\left(\omega_{n}\right) & =\frac{Z_{L 2}^{L}}{Z_{L 1}^{L}} \frac{L-L 1}{L-L 2} \\
& =\frac{\int_{L 2}^{L} w(s) e^{i \omega_{n_{c} \frac{s}{c}}} d s}{\int_{L 1}^{L} w(s) e^{i \omega_{n_{c}}^{s}} d s} \frac{L-L 1}{L-L 2} .
\end{aligned}
$$

Apparently, if $Z_{L 2}^{L} \ll Z_{L 1}^{L}$, and $\epsilon\left(\omega_{n}\right) \approx 0$, the cavity mode at $\omega_{n}$ is resolved; if $\frac{Z_{L 2}^{L}}{L-L 2} \approx \frac{Z_{L 1}^{L}}{L-L 1}$ and $\epsilon\left(\omega_{n}\right) \approx 1$, the cavity mode is not resolved; and if $0<\epsilon\left(\omega_{n}\right)<1$, the cavity mode is partially resolved.

(iii) Resolved impedance calculation with the $Q_{\mathrm{ext}}$ values: Based on the frequencies of the unresolved modes, the cavity mode analysis using an eigenmode solver like OMEGA3P can get the exact $Q_{\text {ext }}$ values of these selected modes. Based on Eqs. (6) and (8), the wake impedances of the unresolved modes can be calculated with the $Q_{\text {ext }}$ values and residual impedance $Z_{L 2}^{L}$ and $Z_{L 1}^{L}$ in the previous step.

Compared to the conventional method, the hybrid method has more superiority in large, complex calculations. There are serious limitations in both conventional time-domain and frequency-domain calculations. The large size significantly increases the simulation time required for direct calculation in time domain, and the complexity of the cavity model dramatically increases the number of cavity modes in frequency domain. Take the APS four-cavity cryomodule as an example. There are hundreds of cavity modes in the APS four-cavity model. Even though most of the modes have very weak coupling with the beam or very low $Q_{\text {ext }}$ values, it is hard to identify which modes should be investigated and which can be neglected. The hybrid method provides a very efficient way to calculate the wake impedance in large, complex problems. With this approach, the simulation time using the time-domain code can be significantly reduced, and it is much more efficient to do cavity mode analysis only for the selected high-Q cavity modes.

The selection of $L$ is a balance of accuracy and efficiency. $L$ is typically set as a value much larger than the beam pipe radius, over $1000 r_{b}$, to ensure that the broadband components has decayed down to a very weak level. A shorter length $L$ makes the time-domain calculation faster, but the background is higher and the spectral resolution is lower, which increases the difficulty to identify the unresolved modes.

As described in the previous analysis, different drive beam schemes are required to calculate the wake in an asymmetric cavity. The single-beam scheme on axis is used to excite the wakes $W_{\|}^{0}$ and $\vec{W}_{\perp}^{0}$. To characterize the transverse wake in an asymmetric cavity, the two-beam excitation scheme is often required for two reasons. First, the two-beam excitation scheme has a dominant dipole moment, while the off-axis one-beam scheme still has a strong monopole moment, which cannot separate the transverse wakes $W_{\perp}^{0}$ and $W_{\perp}^{1}$. Second, in an asymmetric cavity, the transverse wake changes with varying drive dipole directions. And the two-beam scheme has its advantages to investigate this correlation. The dependence of the transverse wake $\vec{W}_{\perp}^{1}$ on the dipole excitation angle $\phi$ is very important in an asymmetric cavity, and it needs to be investigated in detail.

We demonstrate the hybrid method using the APS deflecting cavity as an example. Both the wake impedances of the single cavity and four-cavity cryomodule were investigated using this method.

\section{B. Single cavity wakefield}

In this analysis, the rms length of the Gaussian beams was set as $10 \mathrm{~mm}$, which is the same as the bunch length in the standard operation mode of the APS storage ring. The wakefield up to around $8 \mathrm{GHz}$ were excited. However, the wake impedances above $6 \mathrm{GHz}$, which are well above the beam pipe cutoff frequency of the monopole and dipole mode, were relatively weak and naturally broadened. Thus, we focused on the impedances below $6 \mathrm{GHz}$. During the analysis, we assumed that all propagating fields transmitted through the waveguide and beam pipe could be absorbed without reflection $\left(S_{11}=0\right)$.

We set $L 1=15 \mathrm{~m}$, at which length the broadband impedance had decayed to a relatively weak level. As we will show, we got the resolved impedance based on the wake potential of a length $L=60 \mathrm{~m}$, which was much shorter than the length required in the conventional method. $L 2$ was set as $30 \mathrm{~m}$. The simulation involved direct calculation using GDFIDL and eigenmode calculations using the ACE3P OMEGA3 P solver.

\section{Longitudinal wake}

Figure 10 compares the longitudinal wake impedance $Z_{0 \mathrm{~m}}^{60 \mathrm{~m}}(0 \mathrm{~m}$ to $60 \mathrm{~m})$, residual wake impedance $Z_{15 \mathrm{~m}}^{60 \mathrm{~m}}(15 \mathrm{~m}$ to $60 \mathrm{~m})$ and $Z_{30 \mathrm{~m}}^{60 \mathrm{~m}}(30 \mathrm{~m}$ to $60 \mathrm{~m})$. The impedance instability threshold was roughly estimated based on the instability requirement of the APS storage ring [36].

We can see that the longitudinal impedance is dominated by the $\mathrm{TM}_{010}$-like mode. No other peak appears in the spectrum of the wake impedance $Z_{0 \mathrm{~m}}^{60 \mathrm{~m}}$. However, if we look at the residual impedances $Z_{15 \mathrm{~m}}^{60 \mathrm{~m}}$ and $Z_{30 \mathrm{~m}}^{60 \mathrm{~m}}$, a number of impedance peaks appear. The parameter $e$ of those modes were calculated using Eq. (29), which indicated whether the modes was resolved or not. And then OMEGA3P solver was used to calculate $Q_{\text {ext }}$ values of those unresolved modes. Two transverse modes impedance 


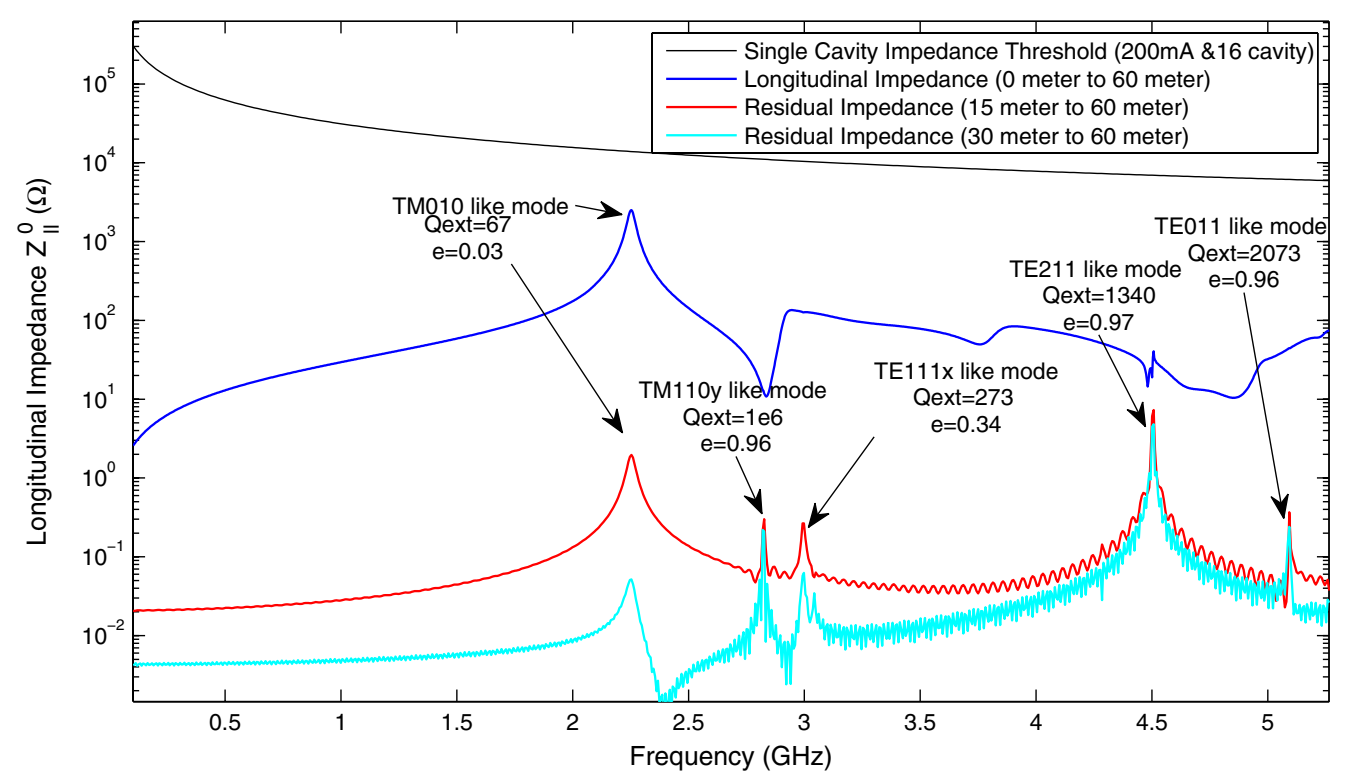

FIG. 10. Single-cavity longitudinal wake impedances $Z_{\|}^{0}$. Three impedance curves are compared: $Z_{0 \mathrm{~m}}^{60 \mathrm{~m}}$ (blue curve), $Z_{15 \mathrm{~m}}^{60 \mathrm{~m}}$ (red curve), and $Z_{30 \mathrm{~m}}^{60 \mathrm{~m}}$ (cyan curve).

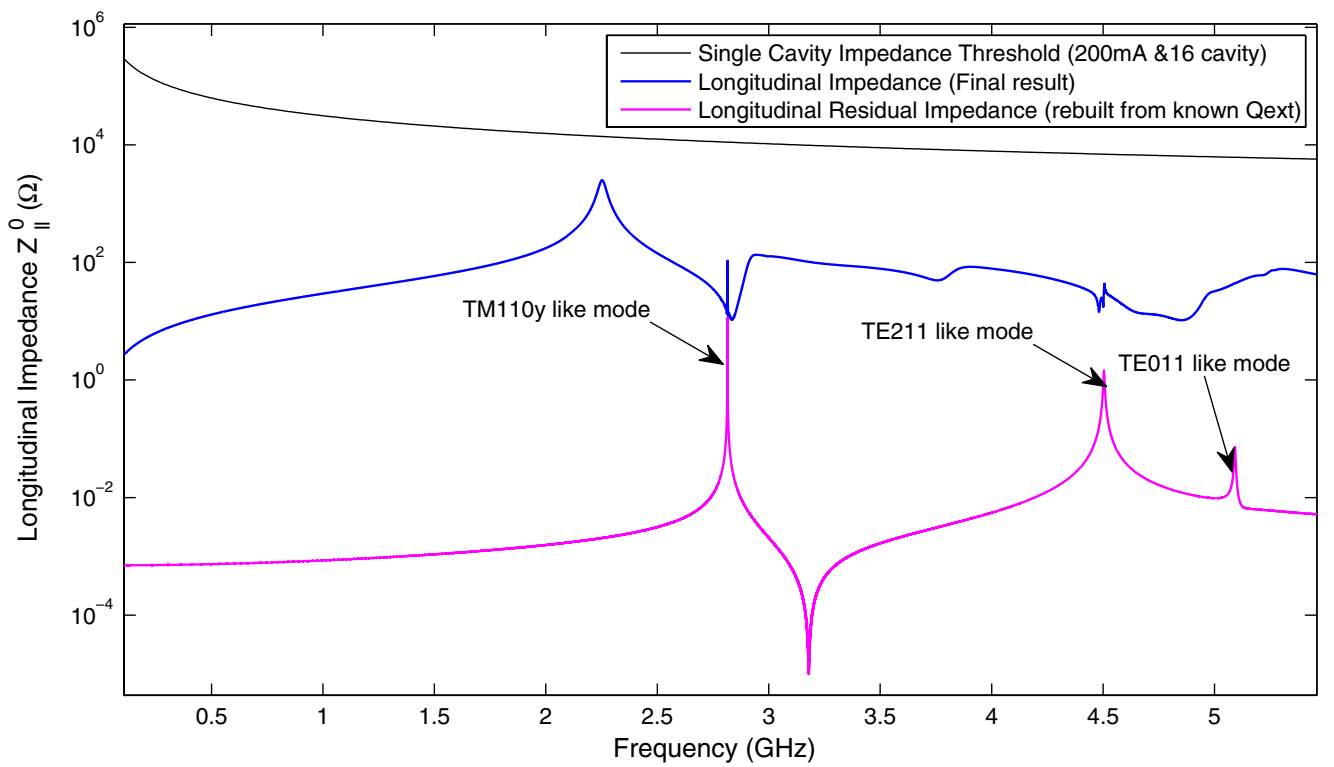

FIG. 11. Two wake impedances are shown in this figure. The pink curve shows the calculated resolved impedances in the frequency domain. The blue curve shows the fully-resolved longitudinal impedance $Z_{\|}^{0}$ based on the $Z_{0 \mathrm{~m}}^{60 \mathrm{~m}}$.

peaks were found in the residual impedance spectrum: the $\mathrm{TM}_{110 y}$-like mode and the $\mathrm{TE}_{111 x}$-like mode. The appearance of these dipolelike modes in the longitudinal spectrum were also due to the asymmetric damping effect. The damper coupling made these transverse modes asymmetric. Thus the beam passing through the cavity center could excite the dipolelike mode. Based on the $Q_{\text {ext }}$ values of the unresolved modes and the residual impedances $Z_{L 2}^{L}$ and $Z_{L 1}^{L}$, we could calculate the resolved impedance without running for hundreds of meters. Figure 11 shows the resolved impedance after taking into account the impedance of the unresolved mode. We can see the sharp impedance peaks of the unresolved modes calculated in the frequency domain.

\section{Transverse wake}

The transverse modes in the APS deflecting cavity have two polarizations in horizontal and vertical planes. In the simulation, the horizontal wake was excited by two beams 


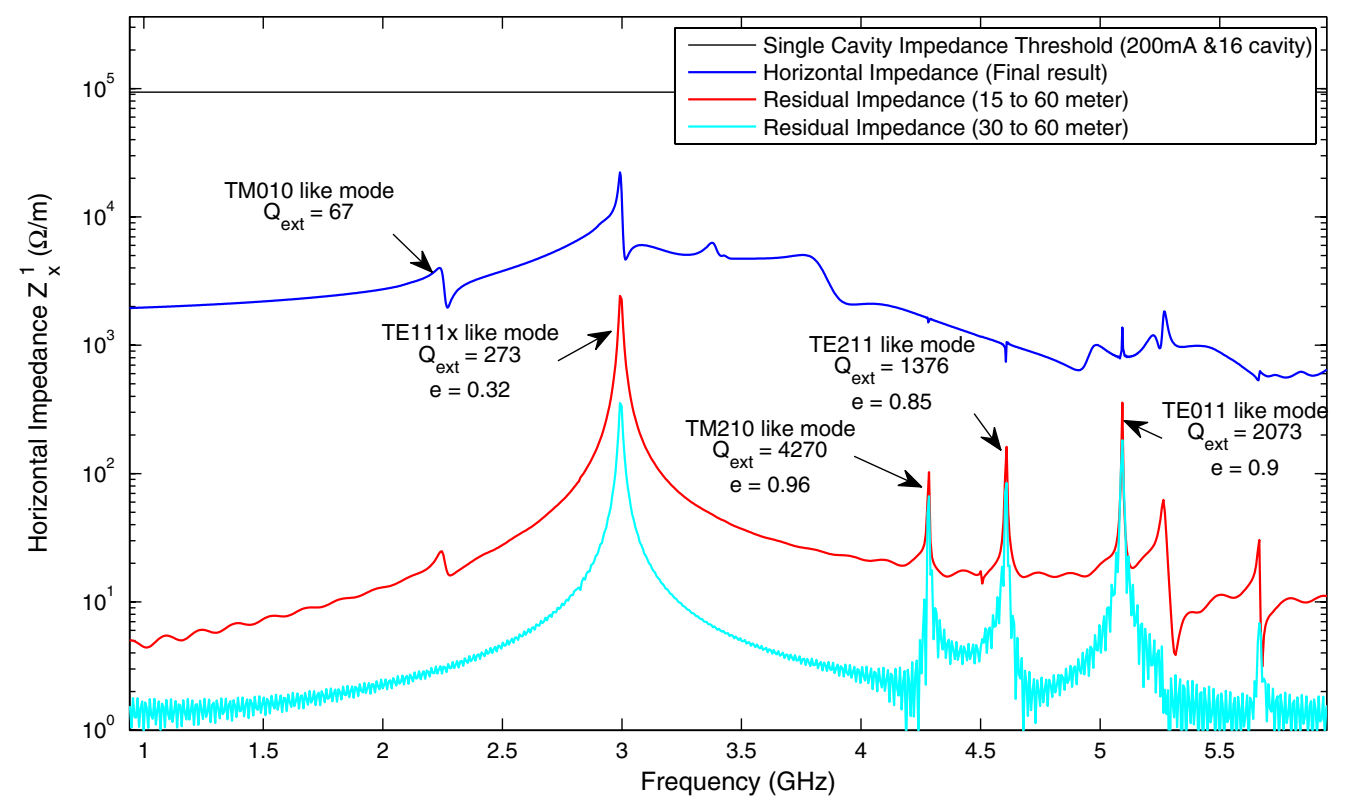

FIG. 12. Single-cavity horizontal wake impedance $Z_{x}^{1}$ and residual impedances. The residual impedances $Z_{15}^{60 \mathrm{~m}}$ (red curve) and $Z_{30 \mathrm{~m}}^{60 \mathrm{~m}}$ (cyan curve) are compared. The blue curve is the fully resolved impedance based on the calculated $Q_{\text {ext }}$ values.

with opposite charges: one with charge $q$ at $(a, 0)$ and the other with charge $-q$ at $(-a, 0)$. The vertical wake was excited in a similar way, with two beams $q$ at $(0, a)$ and $-q$ at $(0,-a)$. To verify the decomposition, the $45^{\circ}$ plane was also investigated with two beams at $(a, a)$ and $(-a,-a)$. In our simulation, $a$ varied from $0.5 \mathrm{~mm}$ to $4 \mathrm{~mm}$ to check the linearity.

Figure 12 compares the transverse impedances. The $\mathrm{TE}_{111-x}$-like mode peak is present in the impedance spectrum. Due to the asymmetry induced by the dampers, the $\mathrm{TM}_{010}$-like mode can be excited by the two beam scheme and can be seen in the spectrum. The residual impedance comparison between the red curve and cyan curve shows that three modes are not resolved within $60 \mathrm{~m}$. The identity and $Q_{\text {ext }}$ values of these unresolved modes were analyzed using OMEGA3P, and the fully resolved wake impedance was obtained, as shown in the blue curve in the Fig. 12.

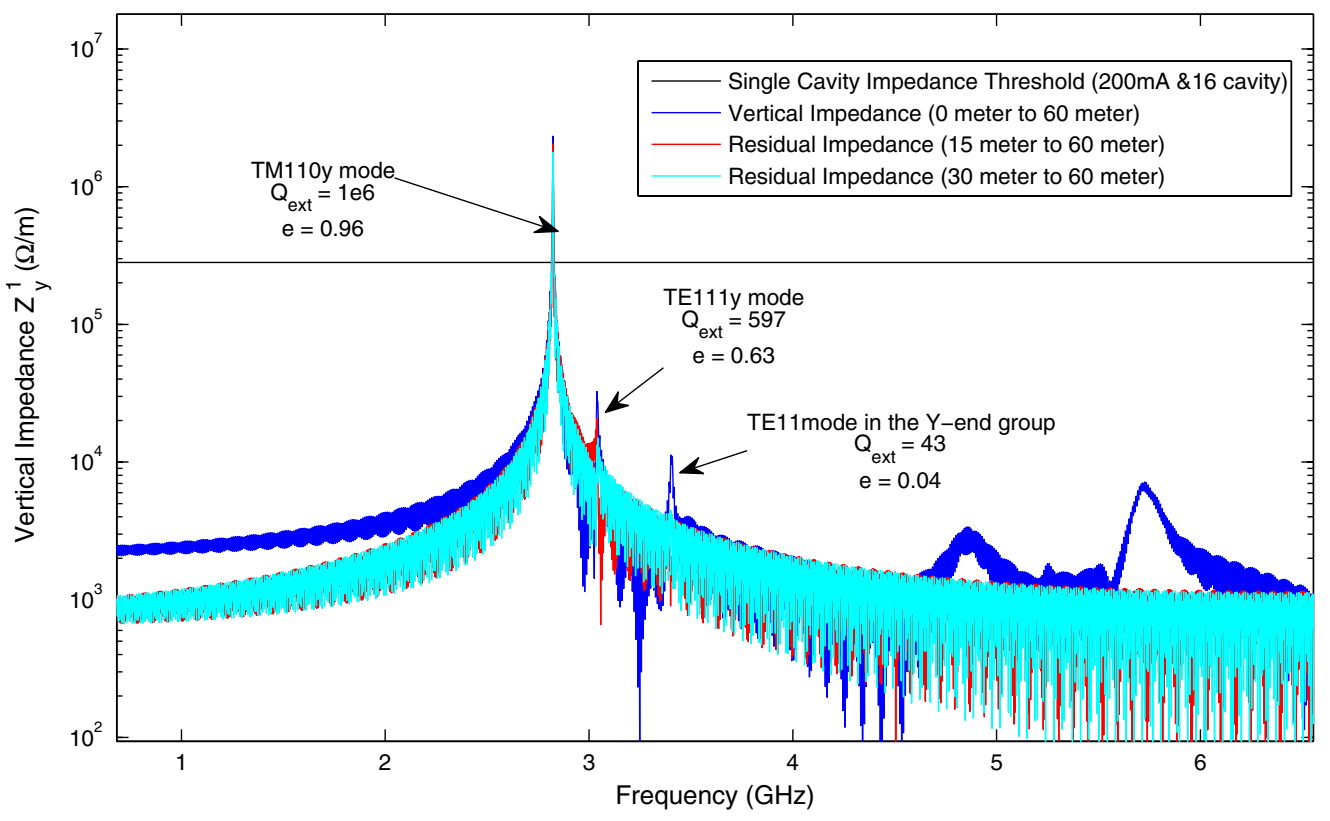

FIG. 13. Single-cavity vertical wake impedances $Z_{y}^{1}$. Three impedance curves are compared: $Z_{0}^{60 \mathrm{~m}}$ (blue curve), $Z_{15}^{60 \mathrm{~m}}$ (red curve), and $Z_{30 \mathrm{~m}}^{60 \mathrm{~m}}$ (cyan curve). 


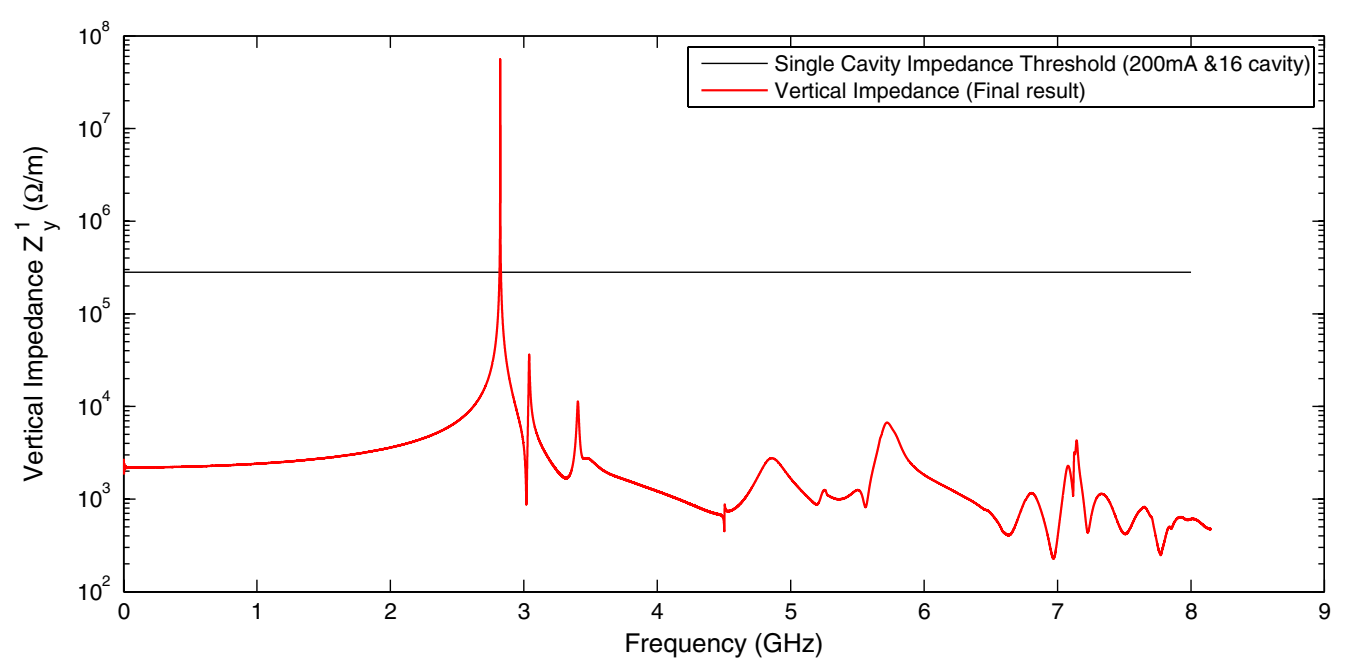

FIG. 14. Single-cavity fully resolved vertical wake impedance $Z_{y}^{1}$ based on the $Q_{\text {ext }}$ values of the unresolved modes.

In the vertical plane, the wake impedance is dominated by the $\mathrm{TM}_{110 y}$-like operating mode. The $Q_{\mathrm{ext}}$ of the operating mode is $1 \times 10^{6}$. The direct calculation used to be very time consuming to get a resolved impedance spectrum, however, the hybrid method can save the simulation time dramatically. Figure 13 shows the impedance spectrum of transverse wake recorded to a length of $60 \mathrm{~m}$. The impedance spectrum has obvious truncation effects mainly due to the operating mode. But we can still use the residual wake comparison to find the mode that is not resolved yet. Note that the residual impedance background is much larger compared with the residual impedance in the horizontal plane. It is possible that there are still unresolved impedance peaks under the background. However, the vertical impedance threshold, which is $3 \times 10^{5} \Omega / \mathrm{m}$, is two orders of magnitude higher than the background. If there is an unresolved impedance peak under the background, the $Q_{\text {ext }}$ value of this mode needs to be higher than $1 \times 10^{6}$ to exceed the instability threshold, which is very unlikely. The residual impedance comparison has shown that at $60 \mathrm{~m}$, there are two modes that are not resolved yet including the operating mode. We obtained the resolved impedance based on the calculated $Q_{\text {ext }}$ values. The resolved vertical impedance is shown in Fig. 14. In this case, the conventional time-domain simulation needs to run up to tens of kilometre to get a resolved spectrum. Using the hybrid method, we only calculated the wakefield for $60 \mathrm{~m}$, which significantly reduced the simulation time. Plus, the $Q_{\text {ext }}$ values calculated by the OMEGA3P solver are more accurate compared with the time-domain calculation [37].

\section{Wakefield coupling between cavities}

For the APS deflecting cavity, four-cavity cyromodules are required to provide the required deflecting voltage of $2 \mathrm{MV}$. Below the beam-pipe cutoff frequency, the unwanted mode was damped by the waveguide damper and the wake impedances were investigated thoroughly, as discussed previously. Above the cutoff frequency, the wakefield might couple with each other through the beam pipe and become trapped between the cavities. The hybrid method helped us a lot in investigating the field coupling between cavities in a two-cavity model as well as in the four-cavity cryomodule model. The two-cavity model is shown in Fig. 15. The interval between cavities is $517 \mathrm{~mm}$, which is the same as the interval in the four-cavity cryomodule.

\section{Longitudinal wake}

Figure 16 compares the longitudinal wake impedances between the two-cavity model and the single-cavity model. Below the beam pipe cutoff frequency, the wake impedance can be regarded as the superposition of two single cavity impedances. Apparently, the impedance spectrum had an oscillating curve right above the TM cutting off frequency. Investigation of these residual impedances clearly shows that a number of new modes appear. Using the OMEGA3P solver, we found these new modes can be classified into two types: the cavity modes and the beam-pipe modes

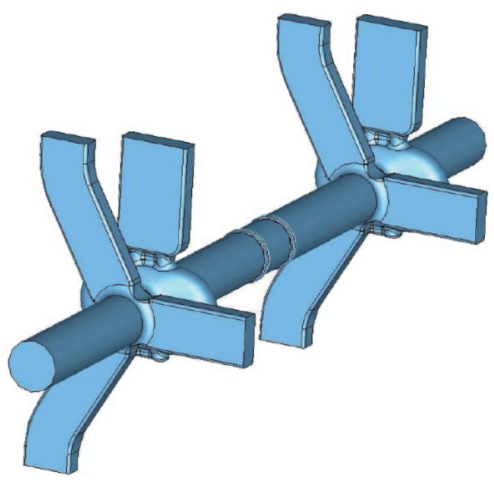

FIG. 15. The two-cavity model used to investigate the wakefield coupling between cavities. The interval between cavities is $517 \mathrm{~mm}$. 


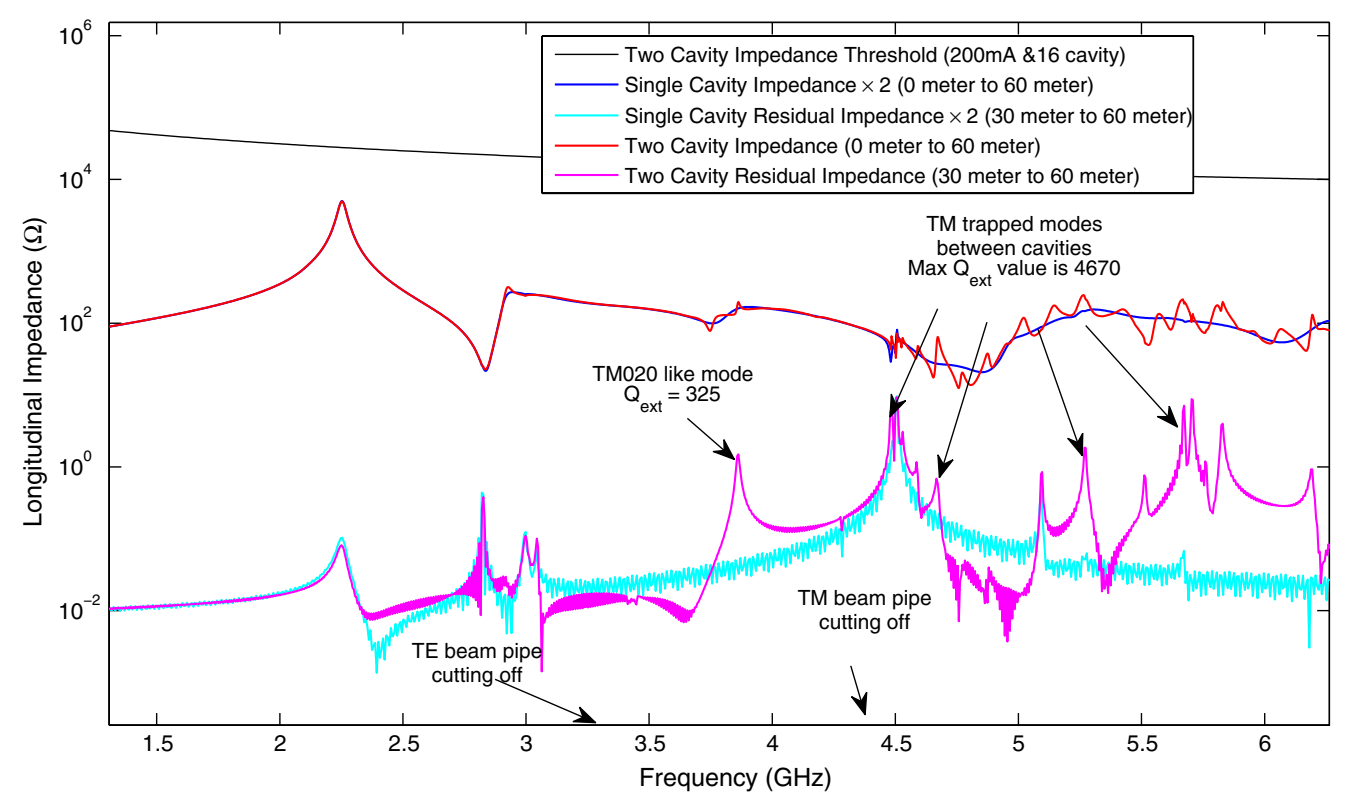

FIG. 16. Longitudinal wake impedance $Z_{\|}^{0}$ comparison between the two-cavity model and the single-cavity model. The impedances are all based on the $L=60 \mathrm{~m}$ wake potentials.

trapped between cavities. An example of the cavity mode is the $\mathrm{TM}_{020}$-like mode around $3.8 \mathrm{GHz}$. The damper has made the mode asymmetric, and this mode has a strong coupling with the beam-pipe TE mode. In the multicavity case, this mode becomes less damped as both ends of the beam pipe between the cavities are connected with the cavity cell. An impedance peak appears in the residual wake spectrum. The trapped modes between cavities are TM modes in the beam pipe, which are the resonant peaks in the residual impedance spectrum above the TM cutoff frequency.

A straightforward and reasonable way to calculate the trapped mode frequency is to treat the beam pipe between the cavity as a resonator with an open boundary at both ends. And the frequencies of the $\mathrm{TM}_{01}$ modes of the beam pipe between the cavity are

$$
\operatorname{fre}_{T M_{01 m}}=\frac{c}{2 \pi} \sqrt{\left(\frac{2.405}{r}\right)^{2}+\left(\frac{m \pi}{L}\right)^{2}}, \quad m=1,2, \ldots
$$

The resonant frequencies estimated by Eq. (30) are quite consistent with the resonance peaks found in residual impedance and cavity mode analyses. Fortunately, the cavity mode analysis has shown that none of them has a $Q_{\text {ext }}$ above 5000, which makes the impedance spectrum well below the instability threshold.

\section{Transverse wake coupling}

The transverse wake coupling between cavities is similar to the longitudinal wake coupling. Figure 17 compares the transverse wake impedance in the horizontal plane between the two-cavity model and the single-cavity model. Above the beam pipe TE cutoff frequency, the cavity impedances start to couple with each other, and the impedance has an oscillating curve. Compared to the longitudinal wake coupling, residual impedance analyses shows that all the $\mathrm{TE}_{11}$ trapped modes are totally or partially resolved at $L=60$ meter, and there is no high- $Q$ mode trapped between cavities.

This difference is caused by the damper property. In the APS cavity design, the trapped mode between the cavities mainly couples with the Y-end group. This damper design tends to have a much stronger coupling with the beam pipe TE mode compared with the TM mode.

Wakefield analysis was also performed on the fourcavity cryomodule. The hybrid method turned out to be a very effective approach to reduce the simulation time and complexity. The direct calculation only recorded the wake potential to a certain length $\mathrm{L}$ (we set it to be $150 \mathrm{~m}$ ) when the broadband impedance and low- $Q$ cavity modes impedance had been resolved. We ran the simulation longer than previous analysis so as to get better spectral resolution of the residual impedance, which helped to better locate the unresolved mode. These unresolved modes were investigated further using OMEGA3P. The number of unresolved modes was only a small part of the total number of cavity modes. In the four-cavity analysis, only 15 impedance peaks were not resolved at a length of $150 \mathrm{~m}$. OMEGA3P could quickly get the $Q_{\text {ext }}$ values of these unresolved modes. The frequencies and the $Q_{\text {ext }}$ values of the unresolved modes in four-cavity cryomodule were generally the same as in the case of the two-cavity model, which corresponded with expectations. In short, the cavity coupling has an effect on the wake impedances above the 


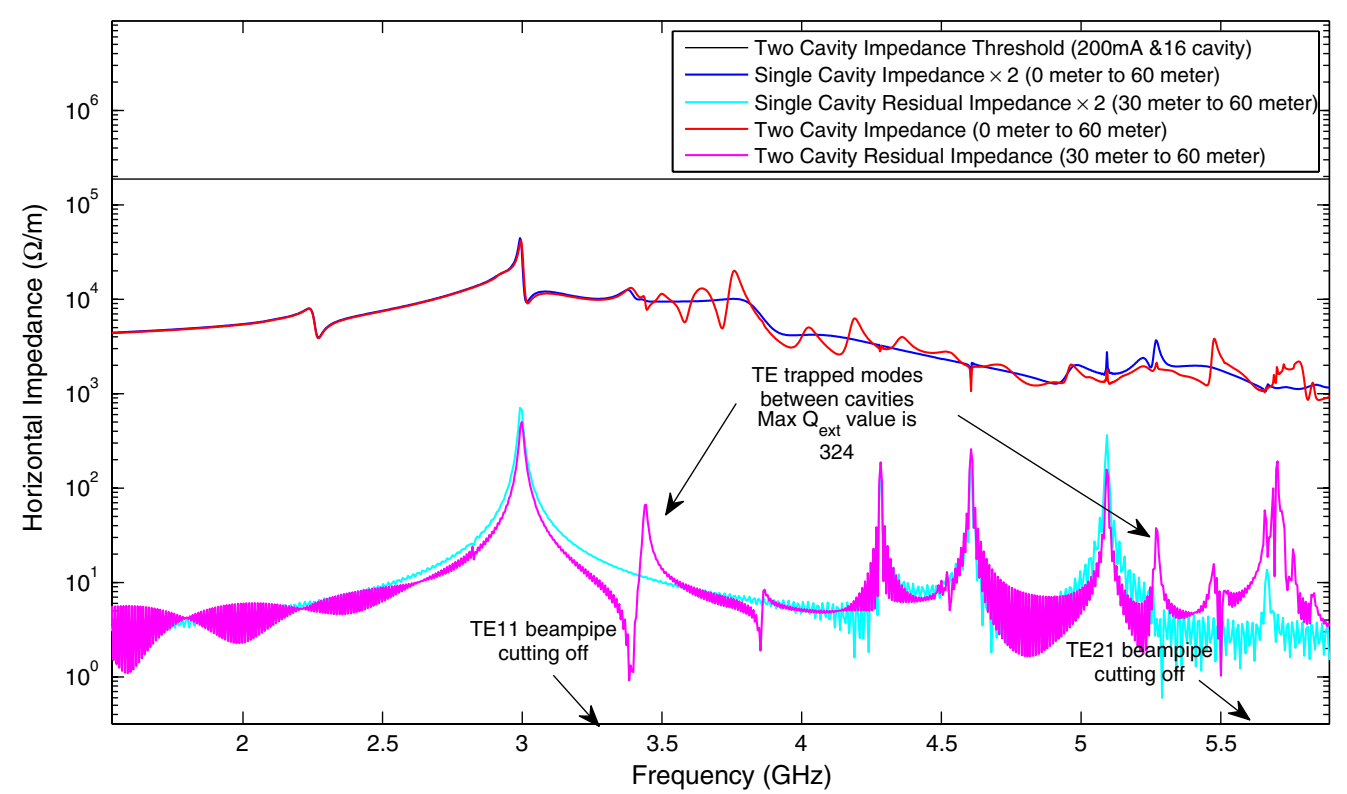

FIG. 17. Transverse impedance $Z_{\perp}^{1}$ comparison between two-cavity model and single cavity model. The impedances are all based on the $L=60 \mathrm{~m}$ wake potentials.

beam-pipe cutoff frequency, but it is unlikely to induce any instability issue. The effects of varying the interval between cavities were also investigated and was found to have a moderate effect on the impedance above the cutoff frequency.

\section{COPPER PROTOTYPE BENCH MEASUREMENT}

To verify the simulation result, bench measurements were performed on a fabricated copper prototype of the APS deflecting cavity. There were two main parts to the measurement: mode measurement and damping measurement. For the mode measurement, we measured cavity mode field distributions along different paths using the Slater perturbation technique. Originally, our plan was to measure the longitudinal and transverse $r / Q$, which required measuring the longitudinal electrical field $E_{z}(r, \theta)$. However, our cavity was relatively compact and it was very difficult to separate the longitudinal and transverse electrical fields in the measurement. After exploring a number of methods, a uniform dielectric bead was selected to measure the electric field amplitude $E_{\mathrm{abs}}(r, \theta)$ rather than the longitudinal component along different paths. If the $E_{\mathrm{abs}}(r, \theta)$ measurement is consistent with the simulation, the $r / Q$ calculation is believed to be accurate.

The mode azimuthal property (monopole, dipole, or higher) is very important to identify the modes, especially in asymmetric cavities. As shown in Fig. 18, bead-pull measurements for each mode were performed with different azimuthal angles-horizontal, vertical, and $45^{\circ}$. This was similar to the wake excitation technique in the wakefield calculations. During the bead-pull measurement, the frequency shift due to the bead perturbation was measured in terms of the phase shift, which was more sensitive than the direct measurement. Before the measurement, a L-band pillbox cavity was used to calibrate the beads. The field distributions of this cavity were well known. Several measurements were performed to get the geometric factor of the beads.

Comparing $E_{\text {abs }}(r, \theta)$ along different beam paths turned out to be very effective in finding the mode identities with the azimuthal property. All the cavity mode measurements showed consistent results with simulation. For example, Fig. 19 compares the measured and simulated field distributions of the $\mathrm{TM}_{010}$-like mode. Both the measured amplitude of the electrical field and the measured gradient

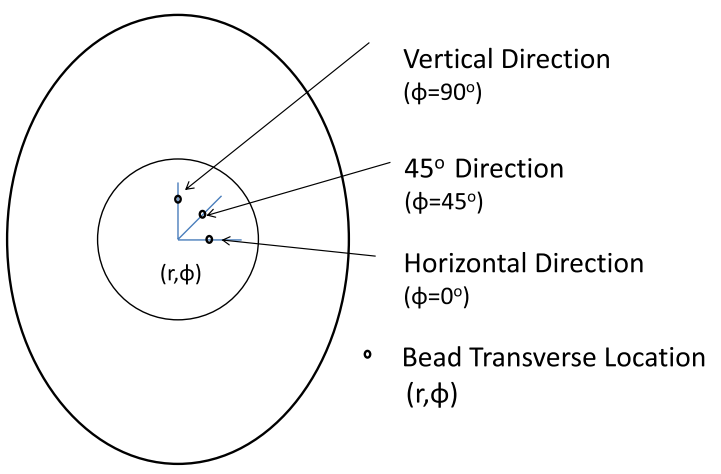

FIG. 18. Bead-pull measurements were performed for each mode at different transverse locations at three azimuthal angleshorizontal, vertical, and $45^{\circ}$ directions. 

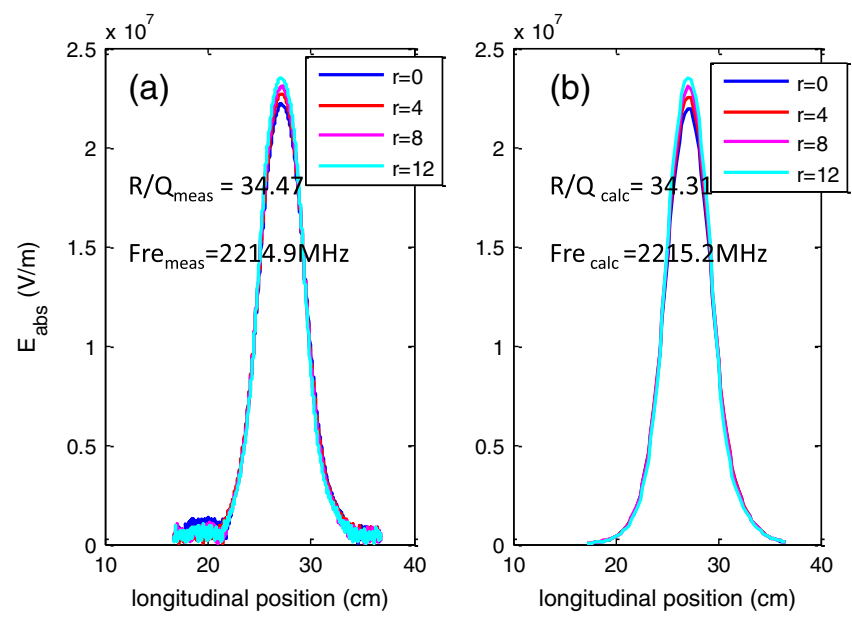

FIG. 19. The measured (a) and calculated (b) field distribution of the $\mathrm{TM}_{010}$-like mode with increasing horizontal offset (on-cell damper directions). The unit of $r$ is millimeter.

along the on-cell damper direction, which caused the transverse kick, were consistent with simulation results.

In the damping measurement, all the waveguide couplers were connected with damper prototypes [38], and $Q$ was measured by the transmission between the probes either inserted into the beam pipe or on the waveguide damper. To get the exact $Q_{\text {ext }}$, we used the difference of the inverse of the measured $Q_{\text {load }}$ values before and after adding the damper to obtain the $Q_{\text {ext }}$. To keep the probe coupling consistent as much as possible, the same type of probes were used with the same mounting location for each mode. If the coupling was not strong enough to get the impedance peak, the probe mounting locations were adjusted until the impedance peak was identified. The $\mathrm{TM}_{110 y}$-like mode $Q_{\text {ext }}$ measurement was different. The coupling of this mode was critical to verify the power coupling. A matched tophat was fabricated to perform this measurement. The transmission between the matched tophat and the tuned beam-pipe probe was directly used to measure the coupling through the power coupler. Table III compares the measured and

TABLE III. Mode frequencies and $Q_{\text {ext }}$ values from measurements and simulations.

\begin{tabular}{|c|c|c|c|c|}
\hline $\begin{array}{l}\text { Mode } \\
\text { identity }\end{array}$ & $\begin{array}{c}\text { Frequency } \\
\text { calculation } \\
(\mathrm{MHz})\end{array}$ & $\underset{\text { calculation }}{Q_{\text {ext }}}$ & $\begin{array}{c}\text { Frequency } \\
\text { measurement } \\
(\mathrm{MHz})\end{array}$ & ${ }_{\text {measurement }} Q_{\text {ext }}$ \\
\hline TM010 ${ }_{\text {like }}$ & 2295.82 & 67.96 & 2294.2 & 76.4 \\
\hline TM110y $y_{\text {like }}$ & 2812.12 & $1 \times 10^{6}$ & 2809.8 & $1.03 \times 10^{6}$ \\
\hline TE111 $x_{\text {like }}$ & 2987.12 & 273 & 2985.23 & 256 \\
\hline TE111y like & 3027.2 & 515 & 3026.74 & 512 \\
\hline TM110 $x_{\text {like }}$ & 3347.4 & 10.2 & 3326.74 & 24 \\
\hline TM020 like & 3937.3 & 200.3 & 3935.4 & 152 \\
\hline TM210 like & 4269.7 & 4270 & 4267.7 & 3890 \\
\hline TE $211_{\text {like }}-1$ & 4505.7 & 1340 & 4520.4 & 1130 \\
\hline TE2 $11_{\text {like }}-2$ & 4599.7 & 1376 & 4610.4 & 1425 \\
\hline
\end{tabular}

calculated frequencies and $Q_{\text {ext }}$ values. It shows that the measured frequency and $Q_{\text {ext }}$ values are quite consistent with the simulation results. More details of the measurement results and techniques are given in [39].

\section{SUMMARY}

Asymmetric cavity geometry has been adopted in a number of superconducting deflecting cavity designs. We investigated how the asymmetric shape affects the wake impedance. The polarization has a significant effect on the transverse wake. And the longitudinal and transverse wake will couple at asymmetric cavity modes. The $r / Q$ values of the cavity modes cannot accurately represent how the wakefield is excited in many asymmetric cavities. Thus, direct calculations in the time domain with different drive beam schemes are required. A set of wake potential and impedance definitions based on the beam moments were used in this paper. Using this definition, we investigate the wake potentials separately with different drive-beam schemes to cover the longrange longitudinal wakefields and transverse wakefields at different azimuthal angles.

Both the time-domain method and the frequency-domain method have their advantages and limitations in wakefield simulation in an asymmetric cavity. An improved method, named the hybrid method, is proposed to take advantage of both the time-domain and the frequency-domain methods. We demonstrated this method using the APS deflecting cavity as a model. In this method, the time-domain calculation is performed to a moderate length where unresolved cavity modes can be selected. Then an eigenmode solver like OMEGA3P can quickly calculate the unresolved $Q_{\text {ext }}$ values in the frequency domain. The fully resolved wake impedances can be calculated without recording the wake potentials for hundreds of meters in the time domain; in the frequency domain, only a limited number of unsolved cavity modes are calculated. This wakefield calculation method is highly useful to improve the efficiency and accuracy for large, complex problems. Using this method, we investigated the coupling between the APS single-cell cavities in the four-cavity cryomodule. We demonstrated that the four-cavity cryomodule of the deflecting cavity could operate in the APS storage ring well below the wake impedance instability threshold.

\section{ACKNOWLEDGMENTS}

We would like to acknowledge useful discussions with Zenghai Li of SLAC. We are particularly grateful to Leonard Morrison, Tim Jonasson, William Yoder, and Charles Montiel for their help in the copper prototype fabrication and bench measurements. This work is supported by the U.S. Department of Energy, Basic Energy Sciences, Office of Science, under Contract No. DE-AC02$06 \mathrm{CH} 11357$. 
[1] R. Palmer, Stanford Technical Report No. SLAC-PUB4707, 1988.

[2] K. Oide and K. Yokoya, Phys. Rev. A 40, 315 (1989).

[3] T. Abe, K. Akai, M. Akemoto, A. Akiyama et al., in Proceedings of the 2007 Particle Accelerator Conference, Albuquerque, New Mexico, USA (2007), p. 27.

[4] A. Zholents, P. Heimann, M. Zolotorev, and J. Byrd, Nucl. Instrum. Methods Phys. Res., Sect. A 425, 385 (1999).

[5] M. Borland, Phys. Rev. ST Accel. Beams 8, 074001 (2005).

[6] M. Placidi, C. Sun, L. Doolittle, P. J. Emma, J. Y. Jung, J. Qiang, and A. Ratti, in Proceedings of the 4th International Particle Accelerator Conference, IPAC-2013, Shanghai, China, 2013 (JACoW, Shanghai, China, 2013), p. 2274.

[7] D. W. Storey, R. E. Laxdal, L. Merminga, and V. Zvyagintsev, in Proceedings of the 25th Particle Accelerator Conference, PAC-2013, Pasadena, CA, 2013 (IEEE, New York, 2013), p. THPBA02.

[8] K. Hosoyama, K. Hara, A. Kabe, Y. Kojima, Y. Morita, H. Nakai, S. Li, K. Ohkubo, H. Hattori, and M. Inoue, in Proceedings of the 1st Asian Particle Accelerator Conference, Tsukuba, Japan (1998), p. 828.

[9] K. Hosoyama, K. Hara, A. Honma, A. Kabe, Y. Kojima, Y. Morita, H. Nakai, K. Nakanishi, K. Akai, K. Ebihara et al., in Proceedings of the 11th European Particle Accelerator Conference, Genoa, 2008 (EPS-AG, Genoa, Italy, 2008), p. 2927.

[10] H. Wang, G. Cheng, G. Ciovati, J. Henry, P. Kneisel, R. A. Rimmer, G. Slack, L. Turlington, A. Nassiri, and G. Waldschmidt, in Proceedings of the 1st International Particle Accelerator Conference, Kyoto, Japan (2010), p. 3061.

[11] G. Waldschmidt, B. Brajuskovic, G. Cheng, J. Mammosser, J. Henry, R. Rimmer, and H. Wang, in Proceedings of the 2011 Particle Accelerator Conference, New York, NY, USA (2011), p. 2516.

[12] R. Calaga, S. Belomestnykh, I. Ben-Zvi, and Q. Wu, in Proceedings of the 3rd International Particle Accelerator Conference, New Orleans, LA, 2012 (IEEE, Piscataway, NJ, 2012), p. 2260.

[13] Z. Li, L. Xiao, C. Ng, and T. Markiewicz, in Proceedings of the 1st International Particle Accelerator Conference, Kyoto, Japan (2010), p. 504.

[14] B. Hall, G. Burt, C. Lingwood, R. Rimmer, and H. Wang, in Proceedings of the 2nd International Particle Accelerator Conference, San Sebastiáán, Spain (EPS-AG, Spain, 2011), p. 322.

[15] S. De Silva, H. Park, J. Delayen, and Z. Li, in Proceedings of the 25th Particle Accelerator Conference, PAC-2013, Pasadena, CA, 2013 (IEEE, New York, 2013), p. WEPAC40.

[16] S. U. De Silva and J. R. Delayen, Phys. Rev. ST Accel. Beams 16, 082001 (2013).

[17] K. Hosoyama, K. Hara, A. Kabe, Y. Kojima, Y. Morita, H. Nakai, K. Saito, T. Furuya, K. Akai, H. Hattori et al., in Proceedings of the 7th Workshop on RF Superconductivity, Saclay, France (1995), p. 671.

[18] G. Burt and H. Wang, Nucl. Instrum. Methods Phys. Res., Sect. A 734, 65 (2014).

[19] G. Waldschmidt, M. Borland, Y. Chae, K. Harkay, D. Horan, A. Nassiri et al., in Proceedings of the 10th
European Particle Accelerator Conference, Edinburgh, Scotland, 2006 (EPS-AG, Edinburgh, Scotland, 2006), p. 3460 .

[20] J. R. Delayen and H. Wang, Phys. Rev. ST Accel. Beams 12, 062002 (2009).

[21] S. De Silva and J. Delayen, Phys. Rev. ST Accel. Beams 16, 012004 (2013).

[22] A. W. Chao, Physics of Collective Beam Instabilities in High Energy Accelerators (Wiley, New York, 1993).

[23] L. Bellantoni and G. Burt, Fermi National Accelerator Laboratory Technical Report No. FERMILAB-TM-2356AD-E-TD, 2007.

[24] R. A. Rimmer, J. M. Byrd, and D. Li, Phys. Rev. ST Accel. Beams 3, 102001 (2000).

[25] D. Li and R. A. Rimmer, in Proceedings of the Particle Accelerator Conference, Chicago, IL, 2001 (IEEE, New York, 2001), p. 915.

[26] F. Marhauser, R. Rimmer, and H. Wang, Thomas Jefferson National Accelerator Facility Technical Report No. JLABTN-08-002, 2008.

[27] T. Weiland, Nucl. Instrum. Methods Phys. Res. 216, 31 (1983).

[28] K. L. Bane, P. B. Wilson, and T. Weiland, in AIP Conference Proceedings, Vol. 127 (AIP, New York, 1985), pp. 875-928.

[29] J. Shi, H. Chen, C. Tang, G. Cheng, G. Ciovati, P. Kneisel, R. Rimmer, G. Slack, L. Turlington, H. Wang et al., in Proceedings of the 11th European Particle Accelerator Conference, Genoa, 2008 (EPS-AG, Genoa, Italy, 2008), p. 913.

[30] L.-Q. Lee, Z. Li, C. Ng, and K. Ko, Stanford Technical Report No. SLAC-PUB-13529, 2009.

[31] G. Wu, H. Wang, C. Reece, and R. Rimmer, in Proceedings of 13th International Workshop on RF Superconductivity, Beijing, China (2007), p. 721.

[32] B. Buckley and G. H. Hoffstaetter, Phys. Rev. ST Accel. Beams 10, 111002 (2007).

[33] http://www.aps.anl.gov.

[34] T. Linnecar, W. Pan, and J. Tuckmantel, CERN Technical Report No. CERN-SL-Note-2001-044-HRF, 2001.

[35] F. Marhauser, R. Rimmer, K. Tian, and H. Wang, in Proceedings of the 23rd Particle Accelerator Conference, Vancouver, Canada, 2009 (IEEE, Piscataway, NJ, 2009), p. 4523.

[36] L. Emery, in Proceedings of the 2007 Particle Accelerator Conference, Albuquerque, New Mexico, USA (IEEE, 2007), p. 4348.

[37] K. Ko, A. Candel, L. Ge, A. Kabel, R. Lee, Z. Li, C. Ng, V. Rawat, and G. Schussman, in Proceedings of the 25th International Linear Accelerator Conference, LINAC-2010, Tsukuba, Japan (KEK, Tsukuba, Japan, 2010), p. 1028.

[38] G. Waldschmidt, B. Brajuskovic, J. Liu, M. Middendorf, A. Nassiri, T. Smith, G. Wu, J. Henry, R. Mammosser, J. Rimmer, and M. Wiseman, in Proceedings of the 3rd International Particle Accelerator Conference, New Orleans, LA, 2012 (IEEE, Piscataway, NJ, 2012), p. 3344.

[39] Y. Yang, A. Nassiri, T. Smith, G. Waldschmidt, H. Wang, and G. Wu, in Proceedings of the 25th Particle Accelerator Conference, PAC-2013, Pasadena, CA, 2013 (IEEE, New York, 2013), p. WEPAC02. 